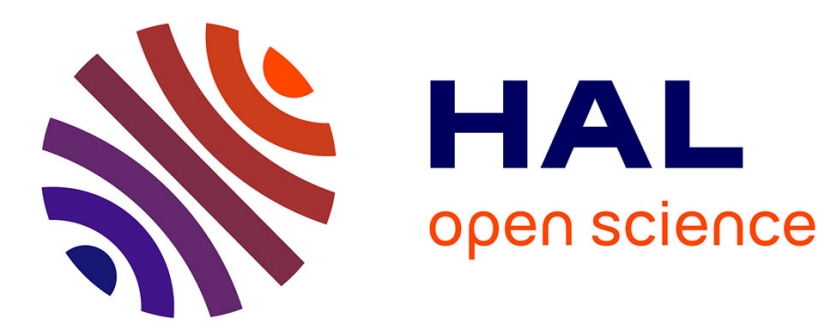

\title{
Influence of mechanical vibrations on quasi-2D silo discharge of spherical particles
}

Arthur Pascot, Naima Gaudel, Sergiy Antonyuk, Jérémy Bianchin, Sébastien Kiesgen de Richter

\section{- To cite this version:}

Arthur Pascot, Naima Gaudel, Sergiy Antonyuk, Jérémy Bianchin, Sébastien Kiesgen de Richter. Influence of mechanical vibrations on quasi-2D silo discharge of spherical particles. Chemical Engineering Science, 2020, 224, pp.115749. 10.1016/j.ces.2020.115749 . hal-02866454

\section{HAL Id: hal-02866454 https://hal.science/hal-02866454}

Submitted on 24 Jan 2022

HAL is a multi-disciplinary open access archive for the deposit and dissemination of scientific research documents, whether they are published or not. The documents may come from teaching and research institutions in France or abroad, or from public or private research centers.
L'archive ouverte pluridisciplinaire HAL, est destinée au dépôt et à la diffusion de documents scientifiques de niveau recherche, publiés ou non, émanant des établissements d'enseignement et de recherche français ou étrangers, des laboratoires publics ou privés.

\section{(ㅇ)(1) $\$$}

Distributed under a Creative Commons Attribution - NonCommercial - NoDerivatives| 4.0 


\title{
Influence of mechanical vibrations on quasi-2D silo discharge of spherical particles
}

\author{
Arthur Pascot ${ }^{\mathrm{a}, *}$, Naïma Gaudel $^{\mathrm{a}}$, Sergiy Antonyuk ${ }^{\mathrm{b}}$, Jérémy Bianchin ${ }^{\mathrm{a}}$, Sébastien Kiesgen De Richter ${ }^{\mathrm{a}, *}$ \\ ${ }^{a}$ Laboratoire d'Energétique et de Mécanique Théorique et Appliquée, Université de Lorraine (UMR 7563 CNRS), 2 Avenue de la \\ Forêt de Haye, 54505 Vandoeuvre-lès-Nancy Cedex, France. \\ ${ }^{b}$ Institute of Particle Process Engineering, University of Kaiserslautern, Gottlieb-Daimler-Str. 44/464, D-67663 Kaiserslautern, \\ Germany
}

\begin{abstract}
In this paper, we present a study of the influence of mechanical vibrations on silo discharges of granular matter. Experimental measurements and discrete element simulations (DEM) are performed in a quasi-two dimensional silo. The influence of both the vibration applied on the entire silo and the opening size on the flow rate is studied. We put in evidence that vibrations allow possible flows at low opening size value, non-existent in the vibration-free case. We also show that vibrations make appear two distinct regimes, governed by both the Froude number $\mathrm{Fr}$ and the relative frequency $\Omega$. In the first regime, a decreased flow rate is observed when increasing the vibration intensity. This behavior is explained by the setting in motion, due to the vibration, of areas previously at rest. In the second regime, DEM simulations evidenced an increased flow rate when increasing the vibration intensity. We find this behavior comes from the intermittent nature of the flow, where the flow rate is directly controlled by the propagation of shock waves all along the silo.
\end{abstract}

Keywords: Silo discharge, Granular matter, Vibration, Rheology

\section{Introduction}

Understanding the rheology and flow properties of granular materials has been a major point of interest in industry. These materials are indeed present in many fields, such as soil studies, medicine and cosmetic manufacturing for example [1]. Industrial processes, which involve granular media, are often based on empirical rules. Among 5 these different industrial applications, a common one is silo discharge.

One of the first study concerning silo discharges was realized by Beverloo et al. [2]. In their work, authors have shown that the flow rate of particles through an orifice mostly depends on the ratio between the opening size and the particle size. Thus, they have proposed an empirical law linking these parameters. Recently, studies have put in evidence limits of this law in particular when the opening size tends to the particle size, and for a silo with a non-zero opening angle $[3,4,5,6]$. In this context, authors $[4,5,6]$ have proposed corrections to the Beverloo law taking into account the decrease of the volume fraction induced by collisions close to the opening. These laws have

\footnotetext{
* Corresponding authors

Email addresses: arthur.pascot@univ-lorraine.fr (Arthur Pascot), sebastien.kiesgen@univ-lorraine.fr (Sébastien Kiesgen De Richter)
} 
been used to successfully predict the silo discharge of complex systems $[6,7,8,9]$. However, these empirical laws fail to predict the clogging of the silo due to the formation of arches at the opening. The probability for these arches to form increases significantly when the opening size becomes close to the particle size $(\sim 5$ particle sizes $)$ $[10,11,12,13]$. This is also the case for more complex particles, where the shape $[14,15,16]$ and surface properties [10] radically change the micro-structure and the contact network between particles. In particular,Govender et al. [16] have shown that arches and clogging are often caused by particle shape, which cannot be captured by rolling friction with spheres, and hence cannot be accurately captured by the Beverloo law. The clogging of silos due to arches formation is often avoided in practical situations, such as industrial silos, by imparting vibrations to the container $[17,18,19,20]$. Previous studies have shown that vibrations allow the flow in normally jammed cases, either due to small opening sizes [18], the silo configuration [21] or the shape of the particles [22].

Recent studies have shown that external vibrations tend to change the apparent rheology of granular materials by suppressing the yield stress $[21,23,24,25,26]$. The granular temperature induces reorganizations of the contact network and an apparent decrease of the viscosity. Some studies have investigated the influence of vibrations on the flow rate in a case of a silo $[27,28]$. They have shown that vertical vibrations tend to decrease the flow rate while horizontal vibrations tend to increase it, depending on the vibration velocity. In a vibrated silo, the flow can be influenced by different parameters: the geometry of the silo (particularly the opening size and angle), the particle properties (density, shape, cohesion) and the vibration parameters (amplitude and frequency). Discrete element simulation (DEM) is a widely used tool in the case of granular media to obtain predictive models, which take into account the influence of these parameters $[7,8,14,22,29,30]$. Moreover, many studies have been conducted to determine the influence of these parameters on the flow rate of a silo [27, 28]. However, it still exists a lack of a general modeling in a wide range of parameters.

In this paper, we present experimental and numerical results of the influence of mechanical vibrations and geometry parameters on the flow rate during silo discharges. In this study, the entire silo is vibrated and non cohesive model granular materials are used. The present article is organized as follows. In Sec. 2 the material and the experimental setup used in the study are presented; the influence of various parameters on the flow rate is presented and discussed. An empirical law taking into account the flow rate, the vibration and geometry parameters is proposed. In Sec. 3 simulation methods are detailed, and experimental results are confronted to numerical ones. Numerical results show a second regime, for which an empirical law is proposed and discussed to describe the evolution of the flow rate.

\section{Experimental measurements}

45 2.1. Experimental methods

The experimental setup (Fig. 1) consists in a rectangular silo made of transparent PMMA sheets with an antistatic coating similar to the one used in Zuriguel et al. [18]. This anti-static coating is regularly applied in order 
to minimize the electrostatic forces and the friction compare to gravity forces. In that case, it has been shown that the wall friction is 1 or 2 order of magnitudes smaller than that due to inelastic collision between particles for dense granular flows [31]. The silo is then filled with spherical and non cohesive glass beads, with a slight dispersion to avoid crystallization and segregation (Silibeads type $\mathrm{S}$, diameter $d=1.1 \pm 0.1 \mathrm{~mm}$ ). The dimensions of the silo and the particle properties are summarized in Tab. 1. On the one hand, the dimensions of the silo ensure a flow primarily 2D (as done in the work of Medina et al. [32]) and allow us to analyze the flow structure in the bulk. On the other hand, the dimensions allow to avoid wall effects before the opening, and an insertion of a number of particles big enough to ensure a steady flow (the silo is filled at $\sim 90 \%$ before each experiment).

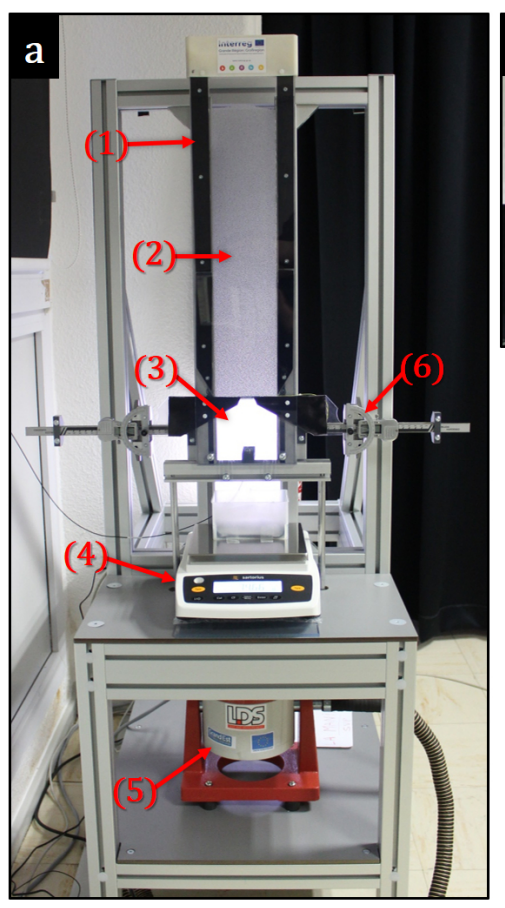

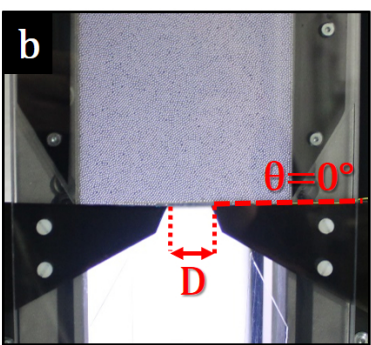

Elements of the setup:

(1) Silo in transparent PMMA with anti-static coating

(2) Particles (1.0-1.25 mm glass beads)

(3) LED Panel (backlight)

(4) Scale (measurement of the mass discharged)

(5) Shaker (apply vertical vibrations)

(6) Opening size (D) and angle $(\theta)$ control

Figure 1: (a) Front view of the experimental setup. (b) Close-up of the silo outlet, with the opening size $D$ and the opening angle $\theta$ ( $D=20 \mathrm{~mm}$ and $\theta=0^{\circ}$ on the picture).

Table 1: Dimensions of the silo and particle properties (glass beads) used in the experiments.

\begin{tabular}{cc}
\hline Parameter & Value \\
\hline$H$ (silo height) & $500 \mathrm{~mm}$ \\
$L$ (silo width) & $100 \mathrm{~mm}$ \\
$W$ (silo depth) & $1.5 \mathrm{~mm}$ \\
$d$ (particle diameter) & $1.1 \pm 0.1 \mathrm{~mm}$ \\
$\rho$ (particle density) & $2500 \mathrm{~kg} \cdot \mathrm{m}^{-3}$ \\
\hline
\end{tabular}

The opening size can be freely adjusted and the silo is linked to a vibration shaker in order to apply vertical and sinusoidal vibrations, with controlled amplitude and frequency values. The tested and available ranges of these four 
parameters are given in Tab. 2. Note that vibrations are limited to acceleration below 33 m.s ${ }^{-2}$ due to both the mass of the silo $(\sim 6 \mathrm{~kg})$ and the shaker limitations $\left(F_{\max }=196 \mathrm{~N}\right)$. The outlet can be manually opened and the particles are collected in a vessel set below the opening. An electronic scale is used to measure the mass discharge with time. The time evolution of the mass discharge is presented in Fig. 2. The discharge reaches nearly instantaneously a stationary flow regime with a constant flow rate, regardless of the parameters used. All subsequent measurements are made in that stationary flow regime.

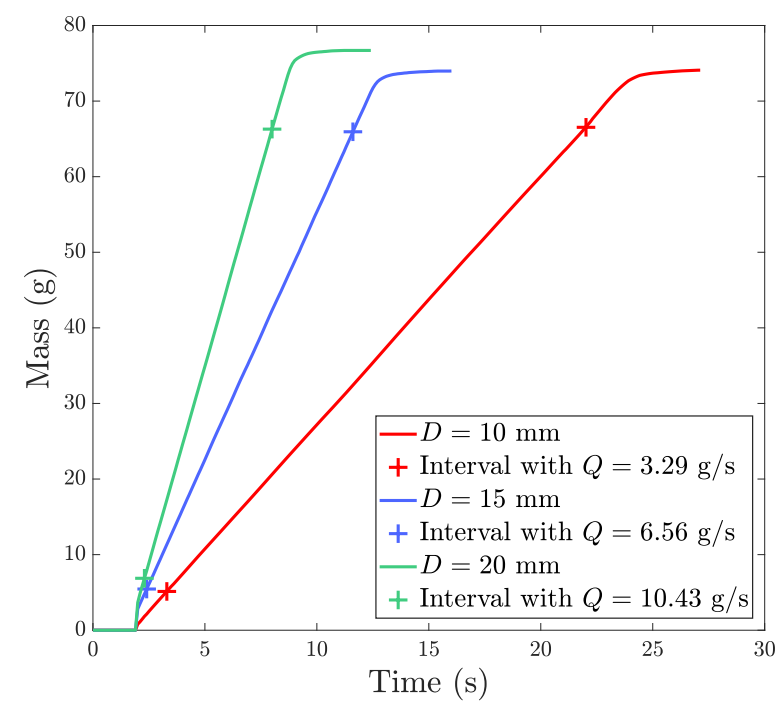

Figure 2: Time evolution of the mass discharge of particles for three different opening sizes, with an acquisition rate of $10 \mathrm{~Hz}$ by using a scale set below the silo. For each case, the corresponding flow rate $Q$ is calculated in the steady state.

Table 2: Vibration and geometrical parameters used in the experiments.

\begin{tabular}{cc}
\hline Parameter & Value/Range \\
\hline$D$ (opening size) & $0-20 \mathrm{~mm}$ \\
$\theta$ (opening angle) & $0^{\circ}$ \\
$f$ (frequency) & $15-75 \mathrm{~Hz}$ \\
$A$ (amplitude) & $0-2000 \mu \mathrm{m}$ \\
\hline
\end{tabular}

\subsection{Experimental results}

65 2.2.1. Flow rate without vibration

Measurements of the flow rate in the vibration-free case are performed and compared to the literature. The flow rate of granular materials through a circular aperture is expected to follow the Beverloo law [2]:

$$
Q=C \rho \phi_{b} \sqrt{g}(D-k d)^{5 / 2}
$$

where $\phi_{b}$ is the bulk volume fraction, $g$ is the gravitational constant, and $C$ and $k$ are the fitting parameters related to a "reduced outlet" due to the presence of particles at the edge of the opening (Fig. 3a). This equation can be 
generalized in the $2 \mathrm{D}$ case $[4,5]$ :

$$
Q=C \rho \phi_{b} \sqrt{g}(D-k d)^{3 / 2}
$$

For a rectangular quasi-2D silo (with $\theta=0^{\circ}$ ), an equation is derived from this law [6]:

$$
Q=C \rho \phi_{b} \sqrt{g} W(D-k d)^{3 / 2}
$$

where $W$ is the depth of the silo.

In our experiments, the bulk volume fraction is experimentally measured when the silo is filled with particles and closed, using image analysis (particle detection). To avoid calculation errors due to particles overlapping (e.g.

$\phi_{b}>1$ ), and to take into account the quasi-2D geometry, the bulk volume fraction is evaluated instead of the purely $2 \mathrm{D}$ surface fraction. We obtain $\phi_{b}=0.47$, which is as expected lower than the random close packing $\left(\phi_{r c p}=0.64\right)$ due to confinement.
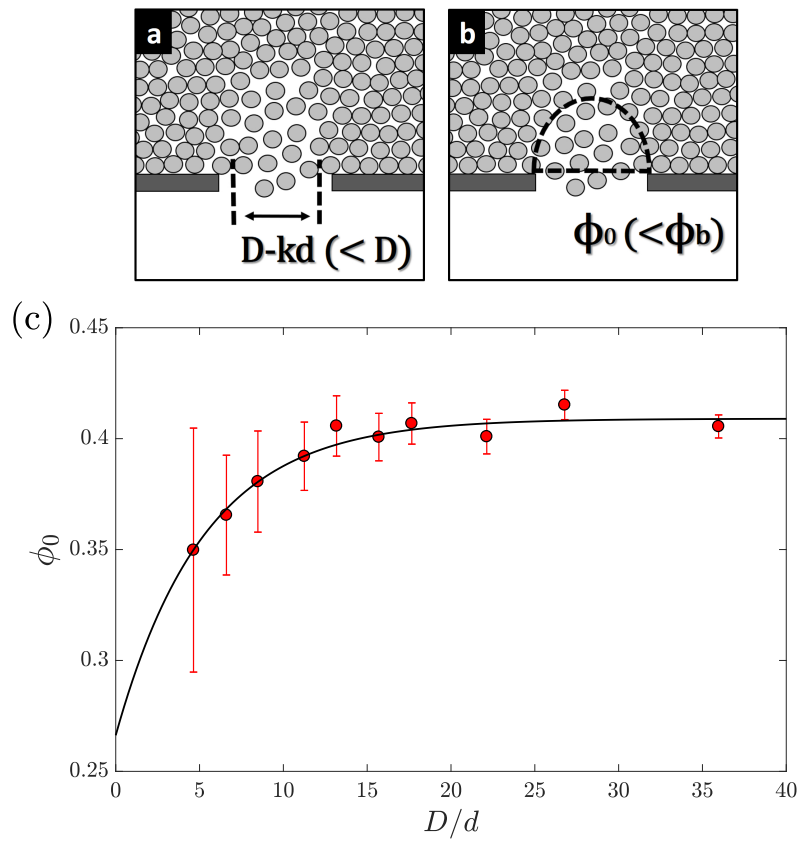

Figure 3: Illustrations of Beverloo's and Benyamine's discharge laws: (a) Existence of a reduced outlet as proposed by Beverloo et al. [2] (Eq. 3) due to the presence of particles at the edges of the opening. (b) Appearance of a dilatency of the volume fraction at the opening as proposed by Benyamine et al. [6] (Eq. 4). (c) Evolution of the volume fraction $\phi_{0}$ obtained experimentally as a function of the dimensionless opening $D / d$. Data are fitted to Eq. 5 (solid line), with $\phi_{b}^{*}=0.41, \alpha=0.349$ and $\beta=0.190\left(R^{2}=0.953\right)$.

The evolution of the flow rate as a function of the opening size is plotted in Fig. 4. The data are well fitted to Eq. 3, which validates the quasi-2D approximation. Note that the R-squared $R^{2}$ statistics are used to evaluate model fit all along the paper ${ }^{1}$. Small discrepancies are observed when $D \rightarrow d$ (Fig. 4, insert), as already reported

\footnotetext{
${ }^{1}$ All fits have been performed using the nonlinear "Levenberg-Marquardt" algorithm.
} 


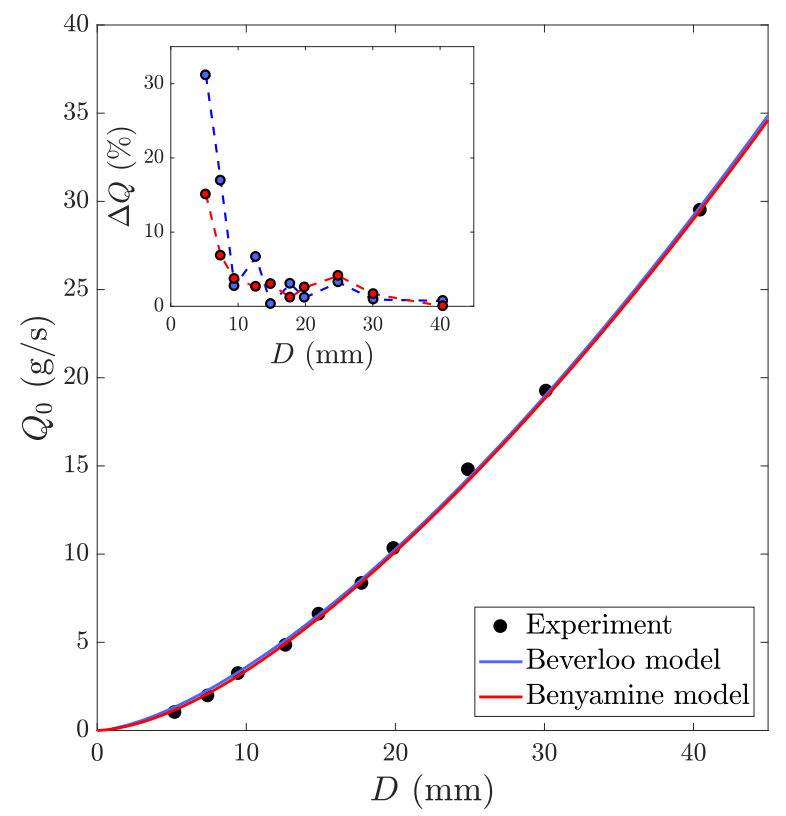

Figure 4: Evolution of the flow rate $Q_{0}$ as a function of the opening size $D$ of the silo. Data are fitted to Eq. 3 from Beverloo et al. [2], with $C=0.665$ and $k=0.113\left(R^{2}=0.999\right)$; and to Eq. 4 from Benyamine et al. [6], with $C=0.735, \alpha=0.349$ and $\beta=0.190\left(R^{2}=0.999\right)$. Insert: evolution of the relative difference of flow rate between the fits and the experimental data, given by: $\Delta Q=\left|\left(Q_{\text {experiment }}-Q_{\text {theory }}\right) / Q_{\text {theory }}\right|$.

in recent studies $[4,5,6]$. These studies propose an exponential correction, related to a decrease of the volume fraction at the opening. In our work, the correction of Benyamine et al. [6] is used:

$$
Q=C \rho \phi_{0} \sqrt{g} W D^{3 / 2}
$$

with $C$ a fitting constant and $\phi_{0}$ the volume fraction at the opening, which results from a dilatency of the flow at the opening (Fig. 3b). $\phi_{0}$ is described by a function $G$, which depends on the particle diameter $d$, the opening angle $\theta$ and size $D[6]$. In our case, for $\theta=0^{\circ}$, it gives:

$$
\phi_{0}=\phi_{b} \times G(D / d, \theta) \underset{\theta=0^{\circ}}{=} \phi_{b} \times\left(1-\alpha e^{-\beta D / d}\right)
$$

where $\alpha$ and $\beta$ are two fitting parameters. This law is known to well predict flow rate for a large range of opening sizes and angles, for monodisperse and polydisperse materials [6]. The evolution of the volume fraction $\phi_{0}$ as a function of the dimensionless opening $D / d$ is plotted in Fig. 3c. The data are well fitted to Eq. 5 , with $\phi_{b}^{*}=0.41$, $\alpha=0.349$ and $\beta=0.190$. Note that the value $\phi_{b}^{*}$ is lower than the value obtained without flow $\phi_{b}=0.47$. A similar result was obtained by Janda et al. [5] where the volume fraction at the center of the opening was lower than the bulk volume fraction $\phi_{\infty}<\phi_{b}$. This discrepancy could also come from the opening size value used $(D=40 \mathrm{~mm})$, which is nearly half the width of the silo.

As presented in Fig. 4, the flow rate data are well fitted to Eq. 4 with only one parameter $C=0.735$. This 
value is in agreement with results obtained by Benyamine et al. [6]. The Benyamine model (4) better captures the behavior for small opening sizes (see Fig. 4, insert). In the following, the flow rate with vibrations $Q$ is normalized by the flow rate without vibration $Q_{0}$ in order to extract the influence of the vibration on the flow.

\subsubsection{Flow rate with vibrations}

The flow rate is measured for four opening sizes when vibrations are applied. For each opening size, experiments are performed for various values of the vibration amplitude while the frequency is kept constant. The frequency $f$ and their corresponding range of vibration amplitude values $A$ used in the experiments are presented in Tab. 3. The vibration parameter is defined as $\Gamma$, the dimensionless acceleration number classically expressed as [18, 33]:

$$
\Gamma=\frac{A \omega^{2}}{g}=\frac{A(2 \pi f)^{2}}{g}
$$

Table 3: Vibration frequencies, amplitudes and corresponding dimensionless accelerations used in the experiments.

\begin{tabular}{ccc}
\hline$f(\mathrm{~Hz})$ & $A(\mu \mathrm{m})$ & $\Gamma$ \\
\hline 15 & $0-2000$ & $0-1.81$ \\
30 & $0-800$ & $0-2.90$ \\
45 & $0-360$ & $0-2.93$ \\
60 & $0-200$ & $0-2.90$ \\
75 & $0-130$ & $0-2.94$ \\
\hline
\end{tabular}

(a) $D=9.47 \mathrm{~mm}$

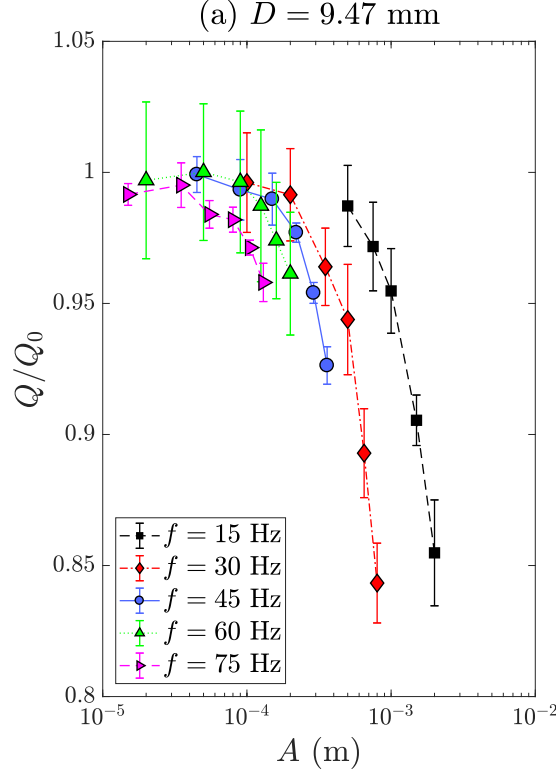

(b) $D=9.99 \mathrm{~mm}$

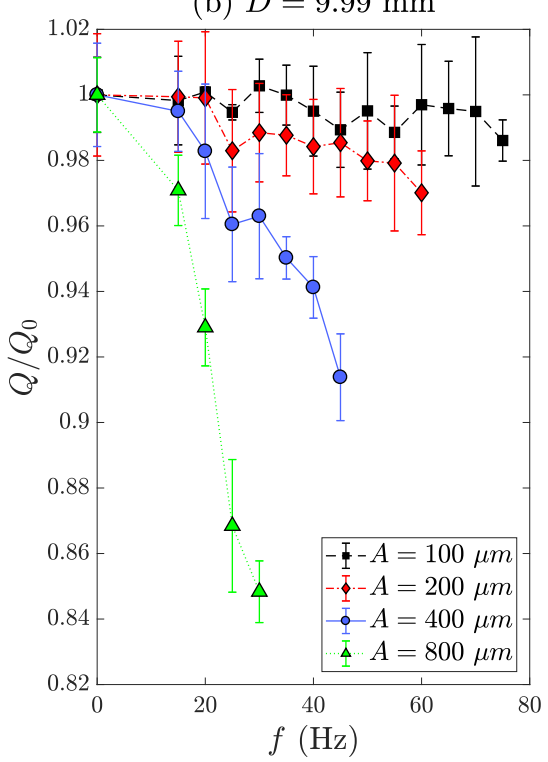

(c) $\Gamma=1.81$

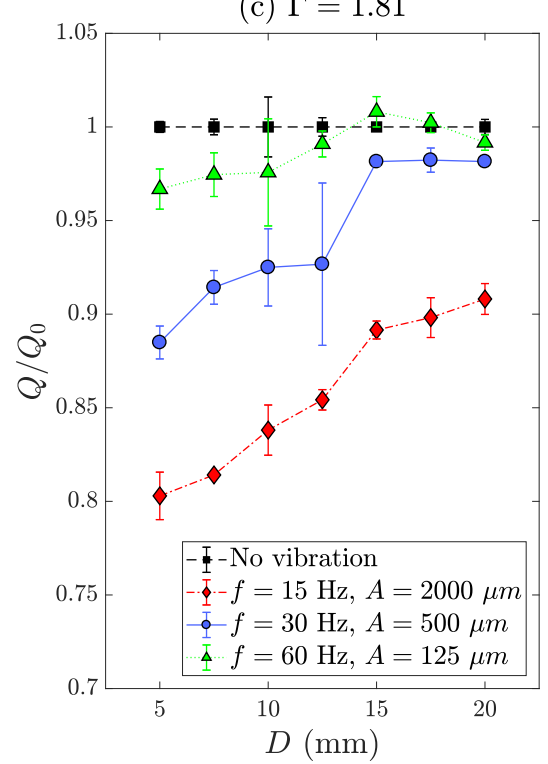

Figure 5: Typical evolution of the normalized flow rate $Q / Q_{0}$ as a function of (a) the vibration amplitude $A$ for different frequency values and an opening size $D=9.47 \mathrm{~mm}$, (b) the vibration frequency for different amplitude values and an opening size $D=9.99 \mathrm{~mm}$, and (c) the opening size $D$ of the silo for different amplitude and frequency values. The $\Gamma$ number is fixed to 1.81 . 
To quantify the effect of vibrations on the flow rate, $Q$ is normalized for each opening size by $Q_{0}$, defined as the flow rate measured without vibration. The evolution of $Q / Q_{0}$ as a function of the opening size $D$, the vibration amplitude $A$ and frequency $f$, is plotted in Fig. 5. The results show that $Q / Q_{0}$ decreases with increasing $A$ and $f$, for a given $D$ value (Fig. 5a and Fig. 5b). However, it increases with increasing $D$ value (Fig. 5c). One can remark that, although the variation of the flow rate observed in our experiments is small $(\sim 20 \%)$, this is significant regarding the very good repetition accuracy (error lower than 5\%).

These results suggest that vertical vibrations lead to the decrease of the flow rate, which depends on $f, A$ and $D$. This behavior is in agreement with the results of Wassgren et al. [28] obtained in a 3D silo configuration with an opening angle $\theta=45^{\circ}$ and a rectangular opening. In Fig. 6, $Q / Q_{0}$ is plotted as a function of the vibration amplitude for all $f$ and $D$ values used in our experiments. The following observations can be made:

- For all opening sizes, the flow rate decreases with the vibration amplitude. The higher the frequency and the opening size, the larger the decrease of the flow rate (black arrow in Fig. 6).

- Regardless the opening size, increasing the vibration frequency seems to start the decrease at a lower amplitude.

- Increasing the opening size seems to reduce the influence of vibration on the flow rate. Indeed, the maximum variation of flow rate shown in Tab. 4 seems to tend to 0 when the opening size increases.

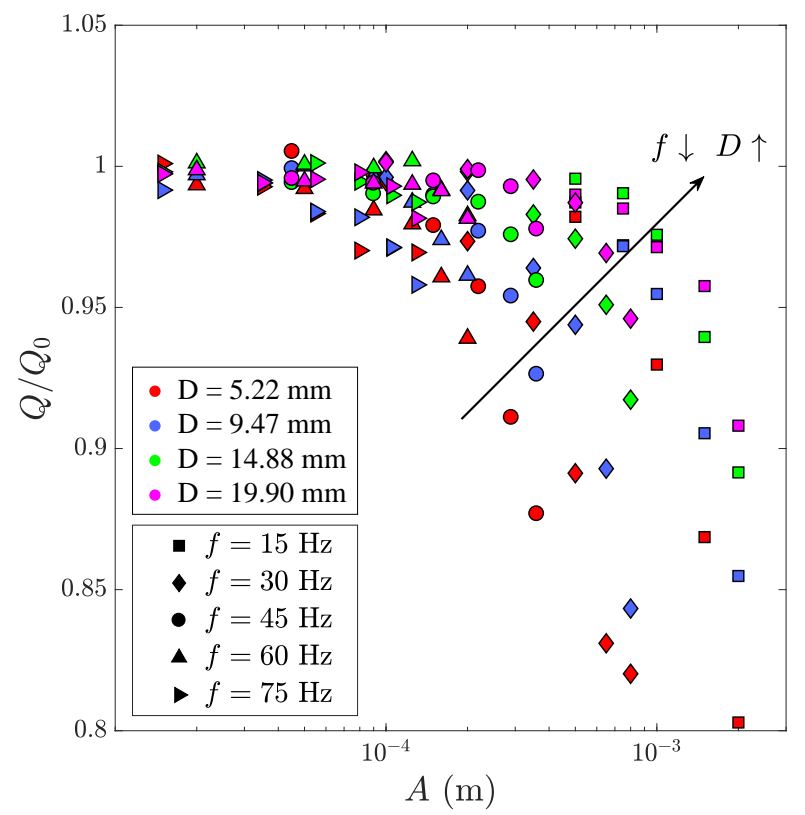

Figure 6: Evolution of the dimensionless flow rate $Q / Q_{0}$ as a function of the amplitude $A$ for all frequency $f$ and opening size $D$ values used in the experiments. 
Table 4: Maximum variation of the flow rate due to vibrations for each opening size. The variation is given by $\delta Q=\left(Q-Q_{0}\right) / Q_{0}$, where $Q_{0}$ is the flow rate without vibration.

\begin{tabular}{c|rrrr}
\hline$D(\mathrm{~mm})$ & 5.22 & 9.47 & 14.88 & 19.9 \\
$\delta Q_{\max }(\%)$ & -19.8 & -15.7 & -10.9 & -9.2 \\
\hline
\end{tabular}

By using PIV measurements, typical flow patterns are extracted when vibrations are applied, as shown in Fig. 7. Increasing the vibration amplitude at a given frequency leads to a transition from a funnel to a mass flow. This behavior can be explained by the setting in motion, due to vibrations, of particles initially at rest in stagnant areas. An increase in vibration amplitude leads to a decrease of the stagnant areas. The consequence of the vibration is essentially the increase of the number of collisions close to the outlet, which decreases the flow rate.
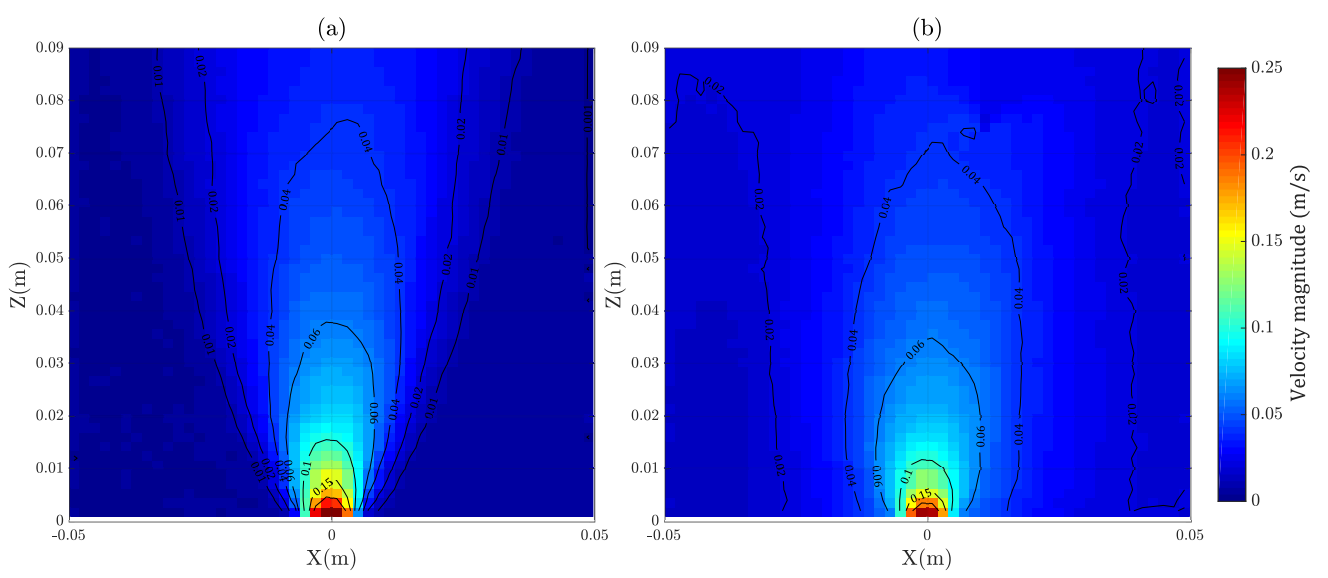

Figure 7: Experimental velocity field at the outlet of the silo for $f=30 \mathrm{~Hz}$ and (a) $A=100 \mu \mathrm{m}$, (b) $A=800 \mu \mathrm{m}$. Black solid lines represent the iso-velocity contours. Two behaviors are observed: (a) a funnel flow and (b) a mass flow at the transition.

\subsubsection{Scaling law}

The evolution of the normalized flow rate $Q / Q_{0}$ is plotted as a function of the Froude number $F r=A \omega / \sqrt{g D}$, as initially introduced by Wassgren et al. [28]. As the configuration studied is quasi-2D, the opening size $D$ is used rather than the hydraulic diameter $\left(D_{h}=\sqrt{D W}\right)$. The data collapse fairly well on a master curve (Fig. 8). Note that a small scattering of the data is observed for the highest vibration amplitude values. As presented in Fig. 8 (solid line), data are well fitted to a simple exponential law, such as:

$$
\frac{Q}{Q_{0}}=\mathcal{F}(F r)=1-a \exp \left(-F r^{*} / F r\right)
$$

where $a=0.421$ and $F r^{*}=0.622$ are two fitting parameters.

This exponential behavior is frequently observed in the dynamics of self-organized complex systems [26, 34] and has to be compared with the transition state approach of Kramers [34]. This analogy is also related to the theory of micro-plasticity where local reorganizations are often described by a Weibull model $\left(1-\exp (t / \tau)^{\beta}\right)$, where 
the exponent $\beta$ equals 1 in most of the observations [35]. In our case, local plastic events in the dead zones are induced by mechanical vibrations that in turn modify the competitiveness level [36] close to the aperture, leading to the decrease of the apparent flow rate. A similar mechanism has been observed in super stable heap (SSH) configurations where granular temperature induced by surface flows creates an exponential profile in the creeping zones [37].

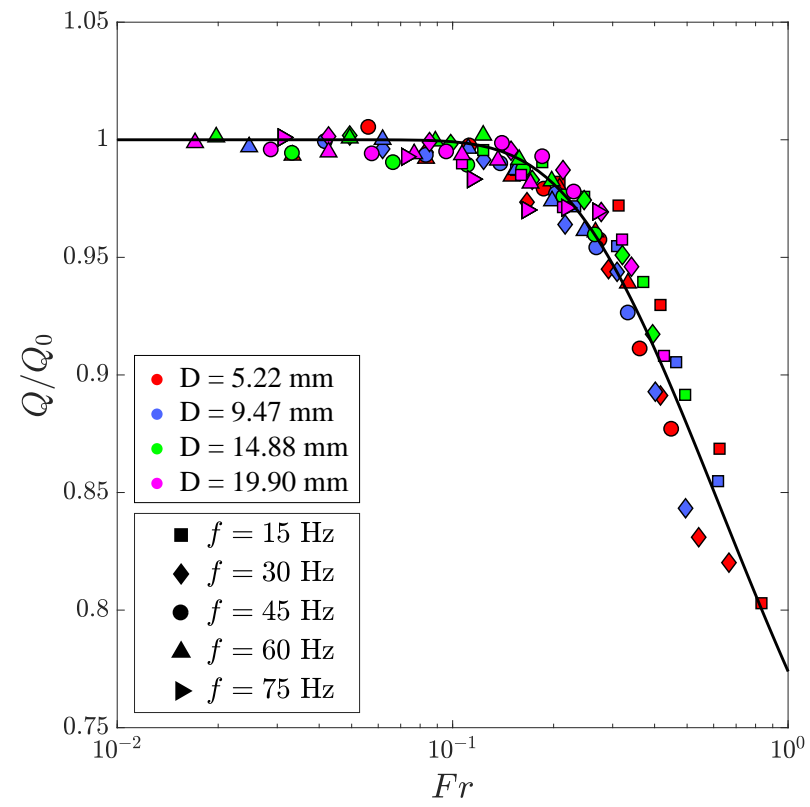

Figure 8: Dimensionless flow rate of the silo $Q / Q_{0}$ as a function of the Froude number $F r$ for various $f$ and $D$ used in the experiments. The data well collapse on a master curve that is fitted to Eq. 7 (solid line), with $a=0.421$ and $F r^{*}=0.622\left(R^{2}=0.941\right)$.

In our work, this law can be interpreted as an activated process induced by vibrations and controlled by the parameter $A \omega / \sqrt{g D}$, which can be interpreted as a Froude number related to the propagation of vibration waves into the system. Moreover, it suggests the existence of a critical Froude number $\mathrm{Fr}^{*}$ close to 1 . For $F r \ll F r^{*}$, the flow rate tends to the flow rate without vibration $Q_{0}$. For $F r \gg F r^{*}$, vibrations significantly modify the flow rate, which should saturate to a limit $Q_{0} \times(1-a)$. This saturation is not observed in our experiments due to experimental limitations to reach high vibration regime, in agreement with a previous study [28]. In order to investigate this behavior for higher $F r$ values, we perform in the next section DEM simulations.

\section{Numerical simulations}

\subsection{Simulation methods}

In this part, discrete element simulations (DEM) are performed using the open-source software LIGGGHTS

$[38,39]$. The model used is based on the work of Cundall and Strack [40], and details are presented in Appendix.

The geometry parameters and the physical properties of particles used in simulations are presented in Tab. 5 . They are similar to the experiments. Typical snapshots of the simulated system is presented in Fig. 9. Simulations 
allow us to investigate flow behavior for high $\Gamma$ values. Thus, simulations are performed for various vibration amplitude and frequency values, i.e. various $\Gamma$ values, also presented in Tab. 5 .
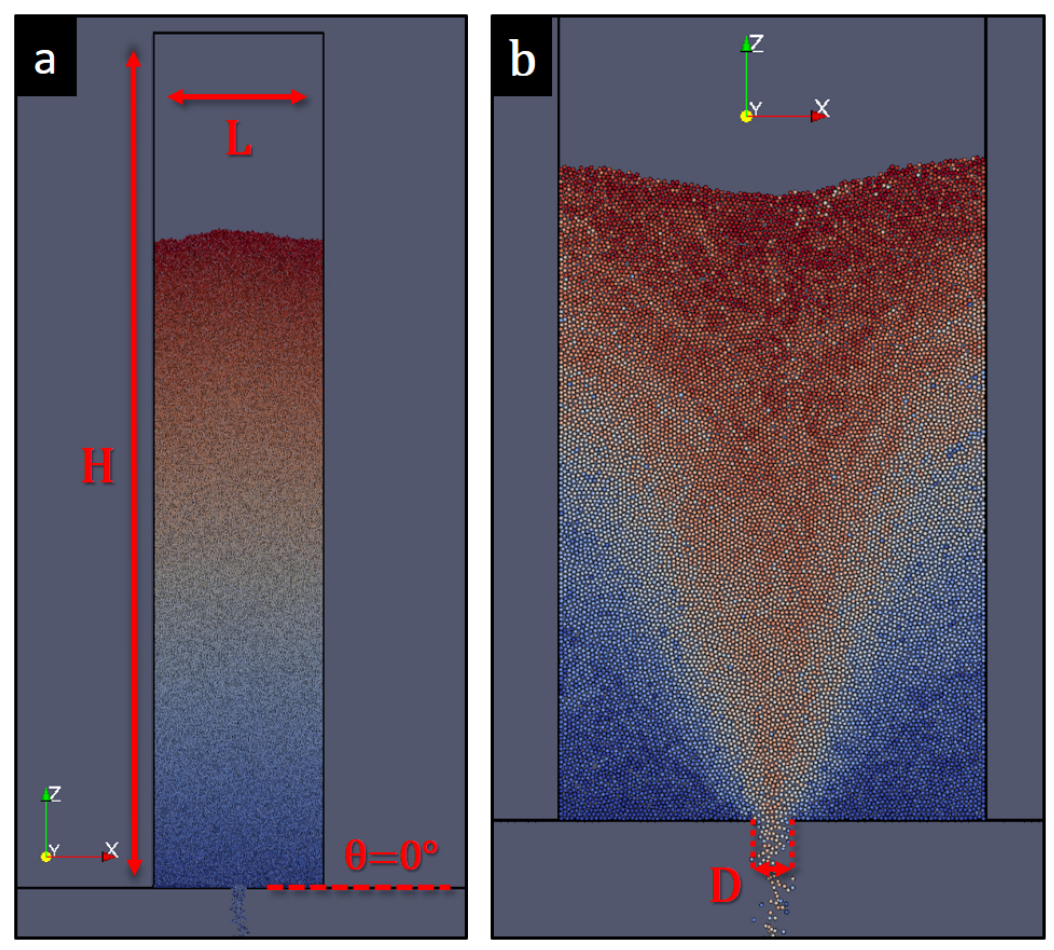

Figure 9: Typical snapshots of the simulated system. Particles are colored in order of insertion, from blue to red. (a) The silo with an opening angle $\theta=0^{\circ}$, a height $H$, a width $L$ and a depth $W$ (values are given in Tab. 5). (b) Particles discharge for $\theta=0^{\circ}$ and an opening size $D=10 \mathrm{~mm}$.

Table 5: Dimensions of the silo, particle and vibration properties used in the simulations.

\begin{tabular}{cc}
\hline Parameter & Value $/$ Range \\
\hline$H$ (silo height) & $500 \mathrm{~mm}$ \\
$L$ (silo width) & $100 \mathrm{~mm}$ \\
$W$ (silo depth) & $1.5 \mathrm{~mm}$ \\
$d$ (bead diameter) & $1.1 \pm 0.1 \mathrm{~mm}$ \\
$\rho$ (bead density) & $2500 \mathrm{~kg} \cdot \mathrm{m}^{-3}$ \\
Number of beads & 40000 \\
$D$ (opening size) & $0-20 \mathrm{~mm}$ \\
$\theta$ (opening angle) & $0^{\circ}$ \\
$A$ (amplitude) & $0-12000 \mu \mathrm{m}$ \\
$f$ (frequency) & $15-75 \mathrm{~Hz}$ \\
$\Gamma$ (relative acceleration) & $0-29.43$ \\
\hline
\end{tabular}




\subsection{Numerical results}

\subsubsection{Flow regimes}

Firstly, the evolution of the flow rate without vibration $Q_{0}$ as a function of the opening size $D$ is plotted in Fig. 10, for both experiments and simulations. Numerical results are in good agreement with experimental ones. Discrepancies are observed for the larger opening sizes and when $D \rightarrow d$ (Fig. 10, insert), where the flow becomes unsteady and difficult to capture numerically. Thus, we have chosen to restrict the numerical study to opening sizes between 10 and $20 \mathrm{~mm}$.

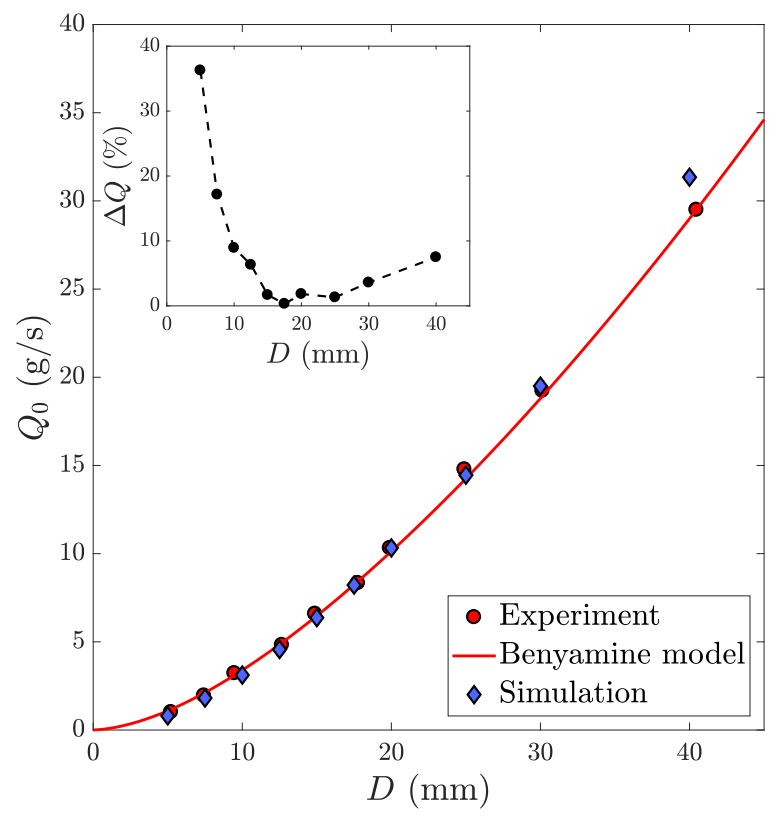

Figure 10: Evolution of the flow rate without vibration $Q_{0}$ as a function of the opening size $D$. Experimental data and Benyamine fit are copied from Fig. 4. Insert: evolution of the relative difference of flow rate between the experimental fit and the simulation as a function of $D$, given by: $\Delta Q=\left|\left(Q_{\text {sim }}-Q_{\text {fit exp }}\right) / Q_{\text {fit exp }}\right|$.

A typical evolution of the normalized flow rate $Q / Q_{0}$ as a function of the vibration amplitude $A$, for $D=10$ $\mathrm{mm}$ and $f=15 \mathrm{~Hz}$, is plotted in Fig. 11a. Results suggest the apparition of two flow regimes. For low $A$ values the flow rate decreases with increasing $A$, in agreement with experiments ( $c f$ Sec. 2.2.2): this is the first regime. Then, the flow rate reaches a minimum before growing up to more than the flow rate without vibration $Q_{0}$ : this is the second regime. 
(a)

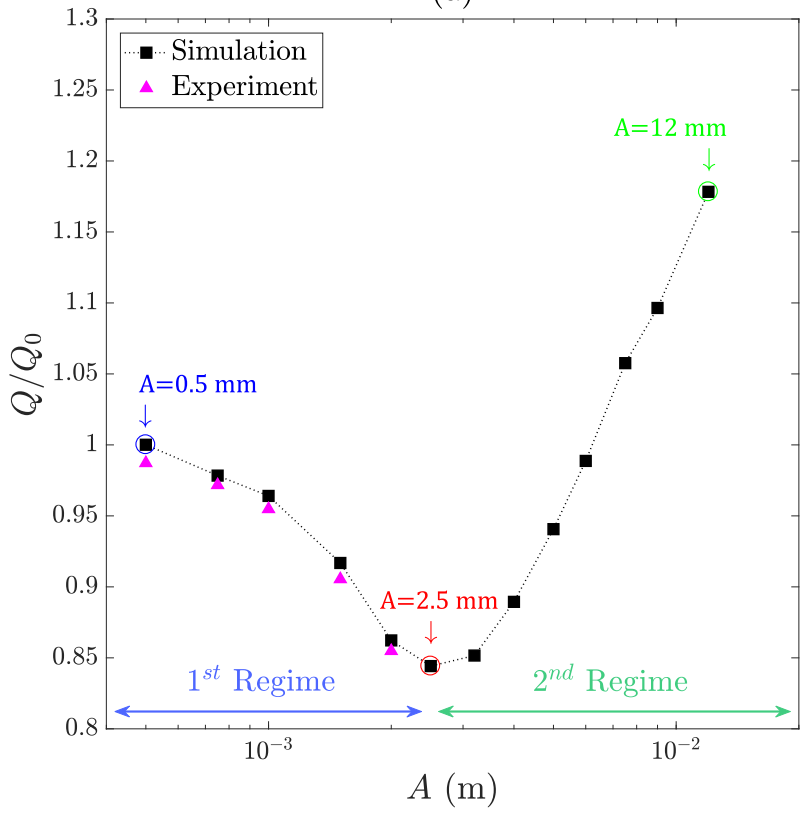

(b)

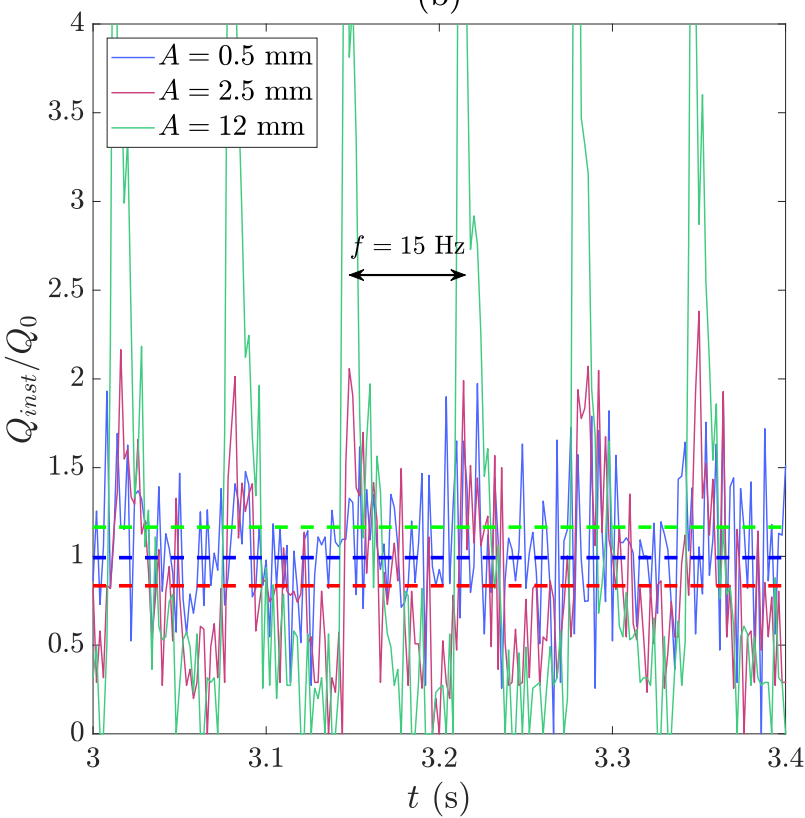

Figure 11: (a) Typical evolution of the normalized flow rate $Q / Q_{0}$ as a function of the vibration amplitude $A$, for $D=10$ mm and $f=15 \mathrm{~Hz}$. (b) Time evolution of the instantaneous normalized flow rate $Q_{\text {inst }} / Q_{0}$, for $f=15 \mathrm{~Hz}$ and for the amplitude values $A=0.5$ mm (blue), $A=2.5 \mathrm{~mm}$ (red) and $A=12 \mathrm{~mm}$ (green). The dashed lines represent the mean flow rate for each amplitude. The instantaneous flow rate is periodic and has the same period as vibrations.

In order to explain this behavior change, the instantaneous flow rate $Q_{\text {inst }}$ is analyzed in each regime. The time evolution of $Q_{\text {inst }} / Q_{0}$, for $f=15 \mathrm{~Hz}$ and for various amplitude values, is plotted in Fig. 11b. To go further, velocity fields obtained numerically in the first regime, close to the transition point and in the second regime are presented in Figs. 12a, b and c; respectively.Note that the numerical velocity fields are in agreement with the ones obtained experimentally (Fig. 7). For each case, the numerical flow patterns during the discharge are presented in Fig. 13. Similar flow patterns are observed in experiment (Fig. 13a and b), though slightly more funneled.

These results put in evidence the existence of a transition from a continuous funnel flow in the first regime to an 155 intermittent flow induced by vibrations in the second regime. As already suggested ( $c f$ Sec. 2.2.2), the continuous funnel flow is explained by the setting in motion, due to vibrations, of particles initially at rest in stagnant areas (Figs. 12a and b, Fig. 13a and b). In the second regime, the increase in flow rate suggests an intermittent burst dynamics due to sudden large rearrangements coming from compression waves propagation all along the packing. This propagation seems to be related to the vibration velocity $A \omega$ (Figs. 11b and 12c). This dynamic is presented in details in Fig. 14. The bursts of instantaneous flow rate are periodic, with the same frequency as the vibration. Moreover, the bursts present a phase shift $\psi=\pi$ compared to the vibration. The steps of this dynamic during one period, presented in Fig. 14, are as follows:

- (1) The silo and the particles near the opening are going down. The particles do not escape the silo and the 
flow rate is zero. Note the presence of an ascending wave $(V z>0)$ from a previous period.

- (2) The silo stops while the particles above the opening are pushed out of the silo by the ones above them, creating a burst of the flow rate.

- (3-4) The silo begins to move upwards and drags the particles along with it. The flow rate decreases.

The size of the bursts should be influenced by the opening size $D$, the gravity $g$ and the vibration velocity $A \omega$. Therefore, the flow rate in the second regime should depends on these parameters, as well as the vibration frequency $f$ since it controls the period of the bursts.

(a)

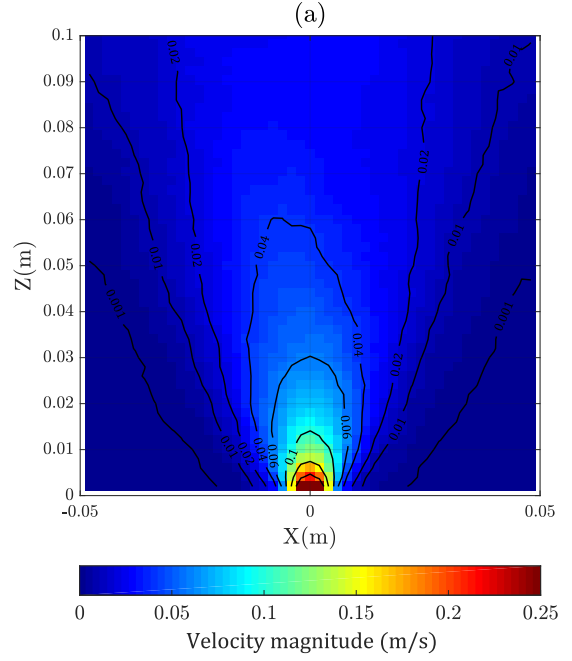

(b)

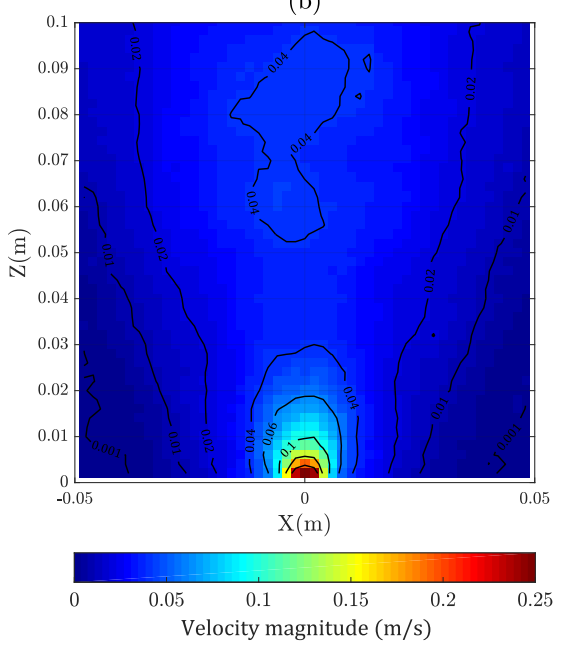

(c)

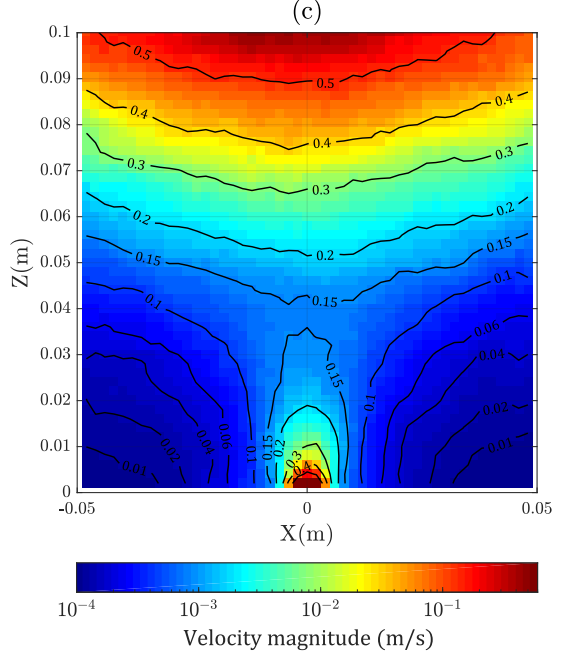

Figure 12: Numerical velocity field at the outlet of the silo for $f=30 \mathrm{~Hz}$ and (a) $A=100 \mu \mathrm{m}$ (first regime), (b) $A=800 \mu \mathrm{m}$ (close to the transition) and (c) $A=8000 \mu \mathrm{m}$ (second regime). Black solid lines represent the iso-velocity contours. Three behaviors are observed: (a) a funnel flow in the first regime, (b) a mass flow at the transition and (c) an intermittent flow in the second regime. 

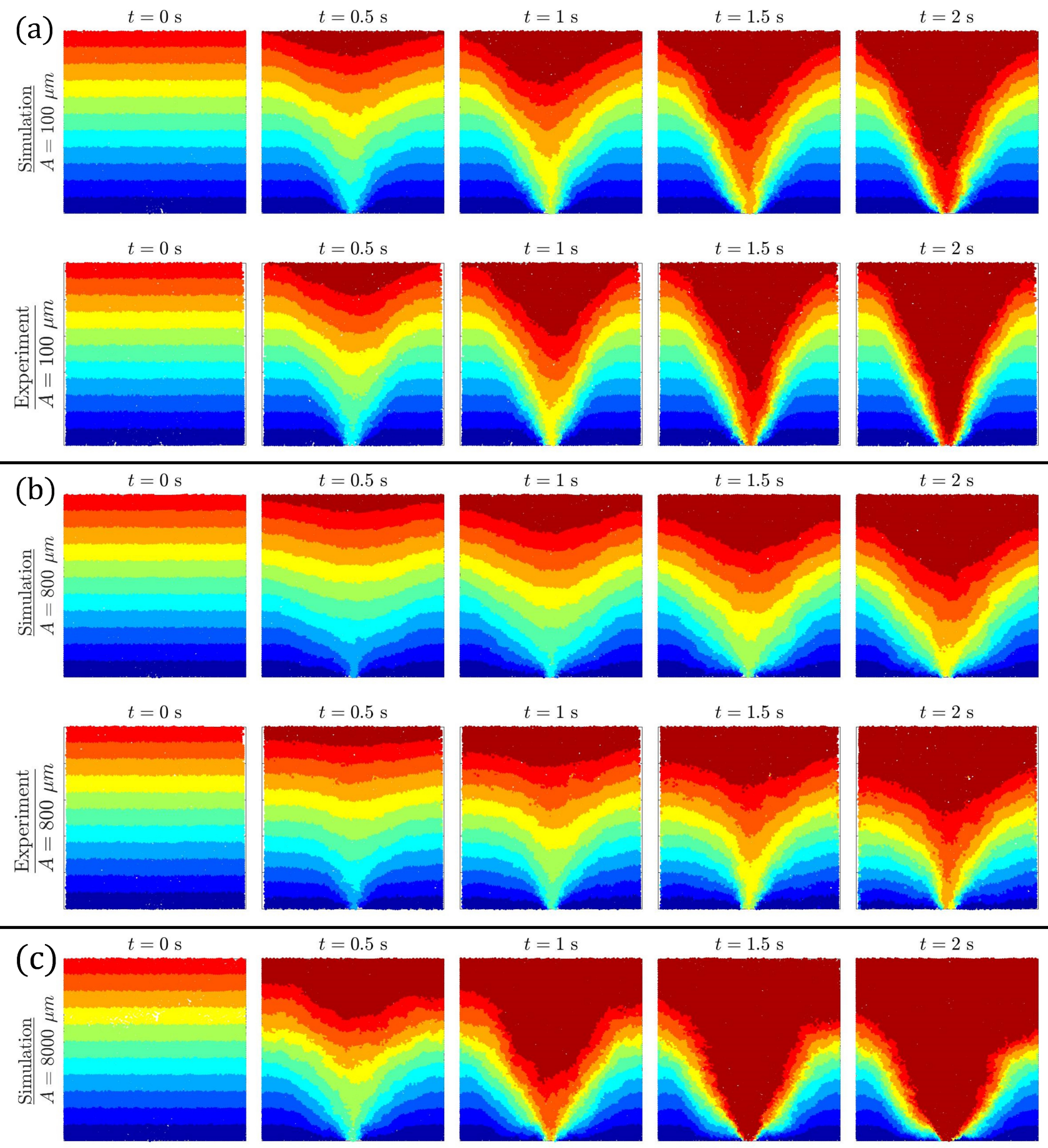

Figure 13: Numerical and experimental flow patterns in the silo during the discharge for $f=30 \mathrm{~Hz}$ and three amplitudes: (a) $A=100$ $\mu \mathrm{m}$ (first regime), (b) $A=800 \mu \mathrm{m}$ (close to the transition) and (c) $A=8000 \mu \mathrm{m}$ (second regime, unattainable in experiment). The particles are colored per layer depending on their initial position, from bottom of the silo (blue) to top (red). 

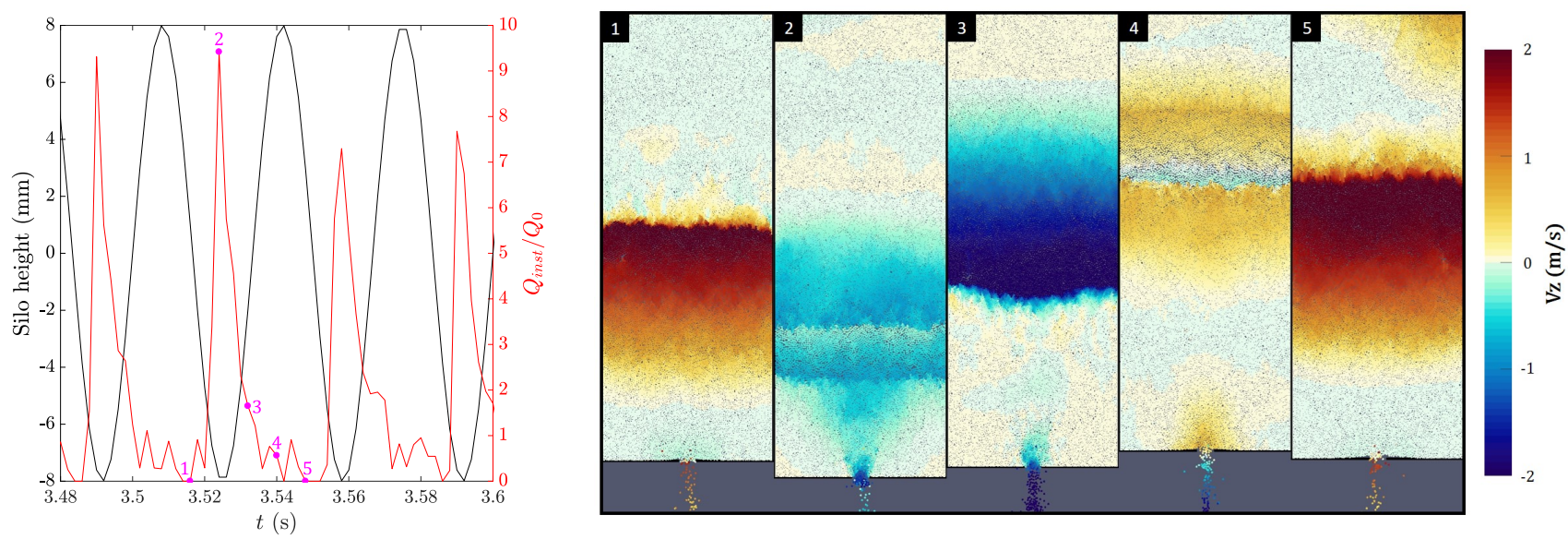

Figure 14: Time evolution of the vertical position of the silo and the normalized instantaneous flow rate during the discharge for $D=10$ $\mathrm{mm}, f=30 \mathrm{~Hz}$ and $A=8000 \mu \mathrm{m}$. For each point 1 to 5 , a snapshot of the flow is presented where particles are colored depending on their relative vertical velocity $V z=V z_{\text {part }}-V z_{\text {silo }}$. The instantaneous flow rate presents periodic bursts (see point 2), with the same period as the vibration and a phase shift $\psi=\pi$.

One can remark that this second regime was not observed by Wassgren et al. [28] in a 3D configuration. In order to determine if the effect of vibrations comes from the confinement, simulations with different wall frictions $\mu_{\text {wall }}$ are performed. The influence of the wall friction on the flow rate for $D=10 \mathrm{~mm}$ and $f=30 \mathrm{~Hz}$ is presented in Fig. 15a. Results suggest that decreasing the wall friction leads to an increase of the flow rate without vibration $Q_{0}$. The dynamics in the first regime is not modified and the wall friction controls the amplitude value at which the second regime appears. The second regime appears at lower $A$ value by lowering the friction. Thus, the results suggest that the second regime can only be observed at very high vibration intensity in $3 \mathrm{D}$ as suggested by the work of Wassgren et al. [28], where the flow seems to start to saturate for high vibration intensity.

Lastly, the influence of the particle density $\rho$ is studied. The effect of the density on the flow rate for $D=10 \mathrm{~mm}$ and $f=30 \mathrm{~Hz}$ is presented in Fig. 15b. The flow rate without vibration is modified as predicted by the Beverloo law [2] and its derived equations (Eqs. 3-4). For the first regime, the effect of the density is negligible. For the second regime, a slight dispersion for the highest amplitude value $A$ is observed. In this case, the dispersion is due to the fluidization of the packing close to the free surface. Our results suggest that the dynamics in both regimes can be considered as independent of particle density. 
(a)

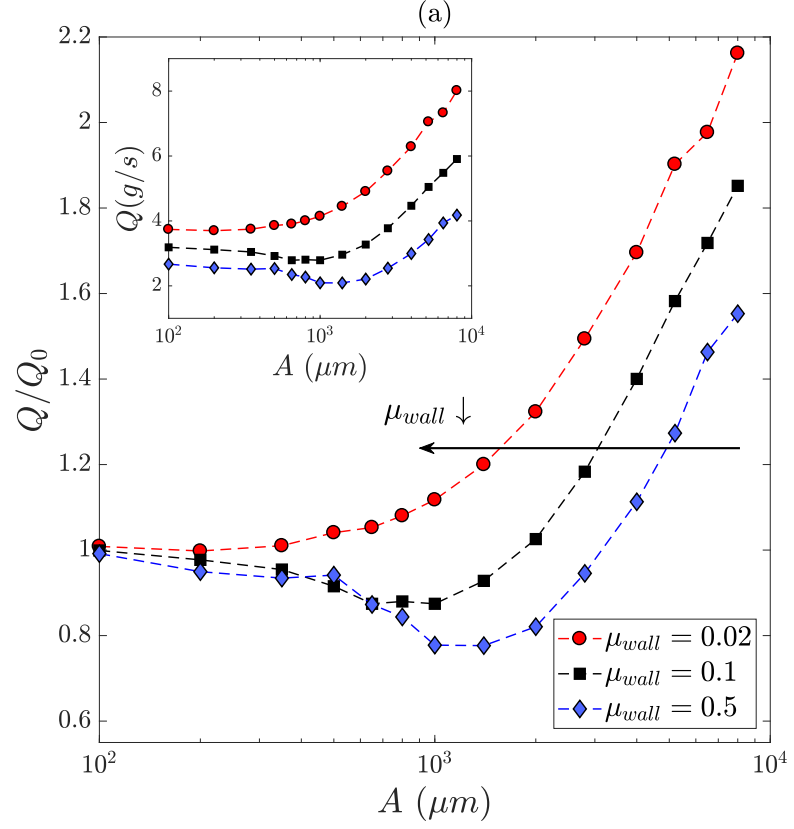

(b)

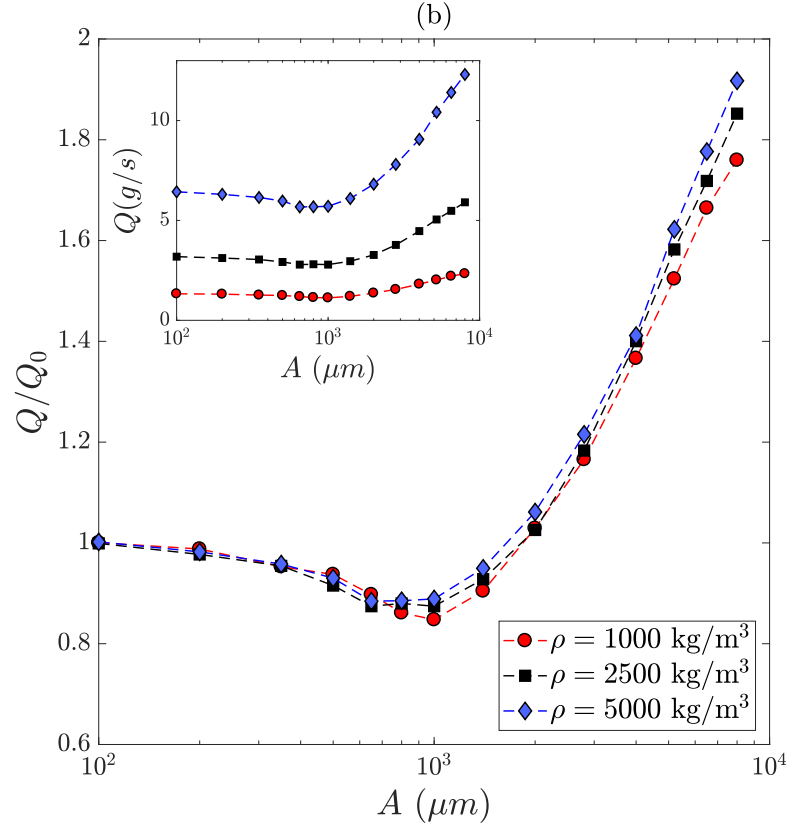

Figure 15: Evolution of the dimensionless flow rate $Q / Q_{0}$ as a function of the amplitude $A$ for $D=10 \mathrm{~mm}, f=30 \mathrm{~Hz}$ and different (a) wall friction values $\mu_{\text {wall }}$ and (b) particle densities $\rho$. Inserts: evolution of the flow rate $Q$ as a function of $A$. In (a) and (b) the reference values used in this paper are colored in black $\left(\rho=2500 \mathrm{~kg} \cdot \mathrm{m}^{-3}\right.$ and $\left.\mu_{\text {wall }}=0.1\right)$.

\subsubsection{Scaling law in the first regime}

The evolution of the flow rate as a function of $D$ is plotted in Fig. 16 in the first regime, for different vibration amplitude, frequency and opening sizes values (Tab. 3). In order to rationalize our data, the evolution of $Q / Q_{0}$ is plotted as a function of Fr, for both experimental and numerical results (Fig. 17a). The data are fitted to Eq. 7. However, some discrepancies are observed at high $F r$ values. Indeed, particles have little time to rearrange between each vibration period at high $F r$ value, i.e. at high frequency. We thus propose to introduce the relative frequency $\Omega$ [41], which takes into account both the typical reorganization time induced by gravity and the period of vibration, such as:

$$
\Omega=\frac{f}{\sqrt{g / d}}
$$

We assume that the relative frequency modifies the exponential term of Eq. 7, in the form $F r \Omega^{\beta}$. A $\beta$ parameter sensitivity analysis has been carried out, and the results suggest that $\beta=1 / 3$. The data are well fitted to a simple exponential law (Fig. 17b) similar to Eq. 7 [6], such as:

$$
\frac{Q}{Q_{0}}=\mathcal{F}_{1}\left(F r \Omega^{1 / 3}\right)=1-a_{1} \exp \left(\frac{-b_{1}}{F r \Omega^{1 / 3}}\right)
$$

where $a_{1}$ and $b_{1}$ are two fitting parameters. Numerical data are well fitted to this equation with $a_{1}=0.522$ and $b_{1}=0.420$. To go further, the evolution of $Q / Q_{0}$ is plotted as a function of $F r \Omega^{1 / 3}$ for both experimental and numerical results (Fig. 18). All the data well collapse on a master curve, which is fitted to Eq. 9 with $a_{1}=0.539$ and $b_{1}=0.438$. 

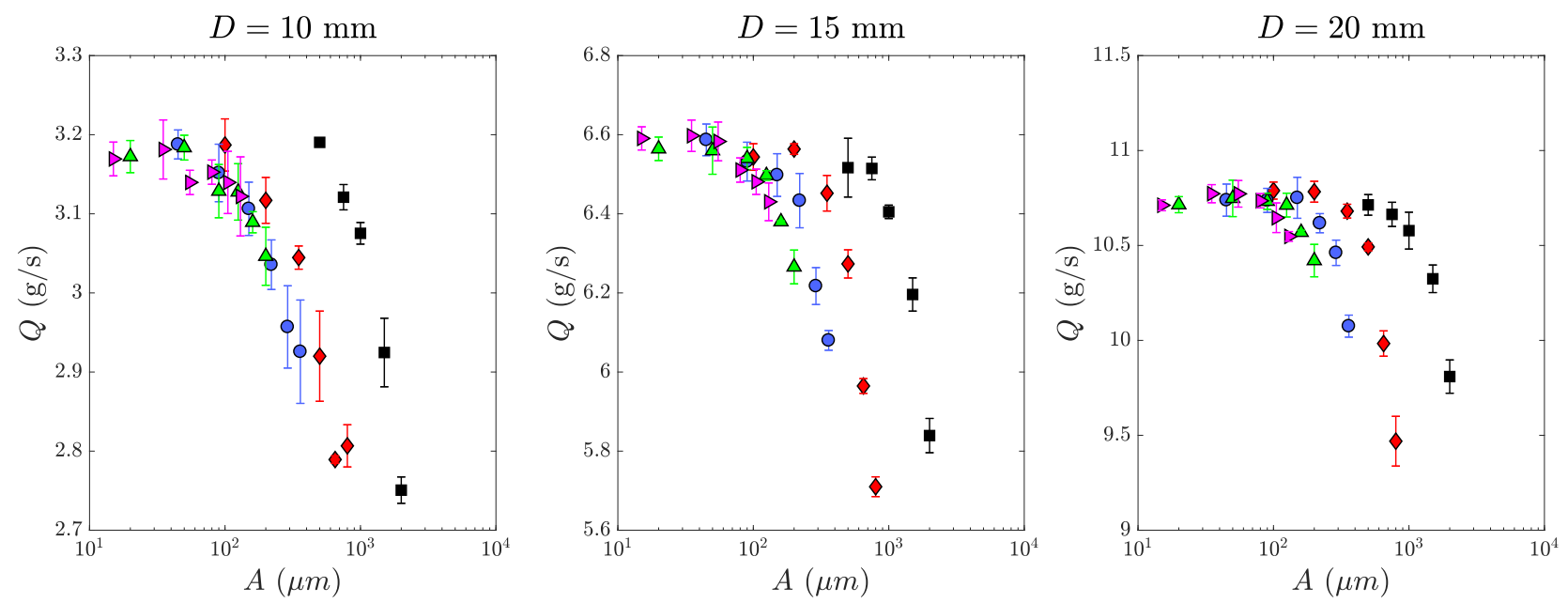

If $f=15 \mathrm{~Hz} \quad$ \ $f=30 \mathrm{~Hz} \quad$ \ $f=45 \mathrm{~Hz} \quad$ \ $f=60 \mathrm{~Hz} \quad$ \ $f=75 \mathrm{~Hz}$

Figure 16: Evolution of the flow rate $Q$ as a function of the vibration amplitude $A$, for various frequency values $f$. Simulations are performed for three opening sizes $D=10,15$ and $20 \mathrm{~mm}$.

(a)

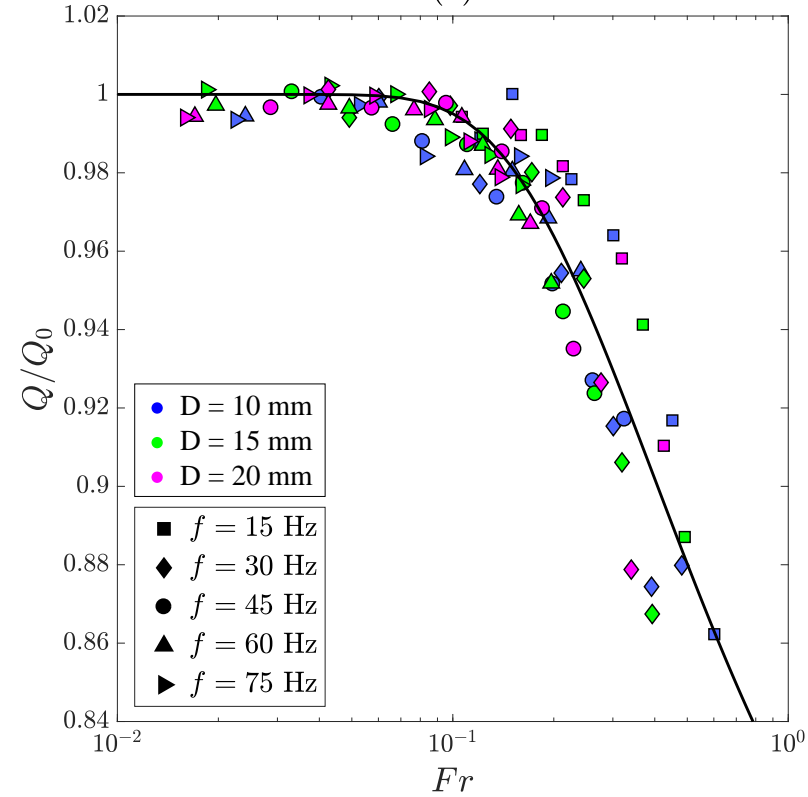

(b)

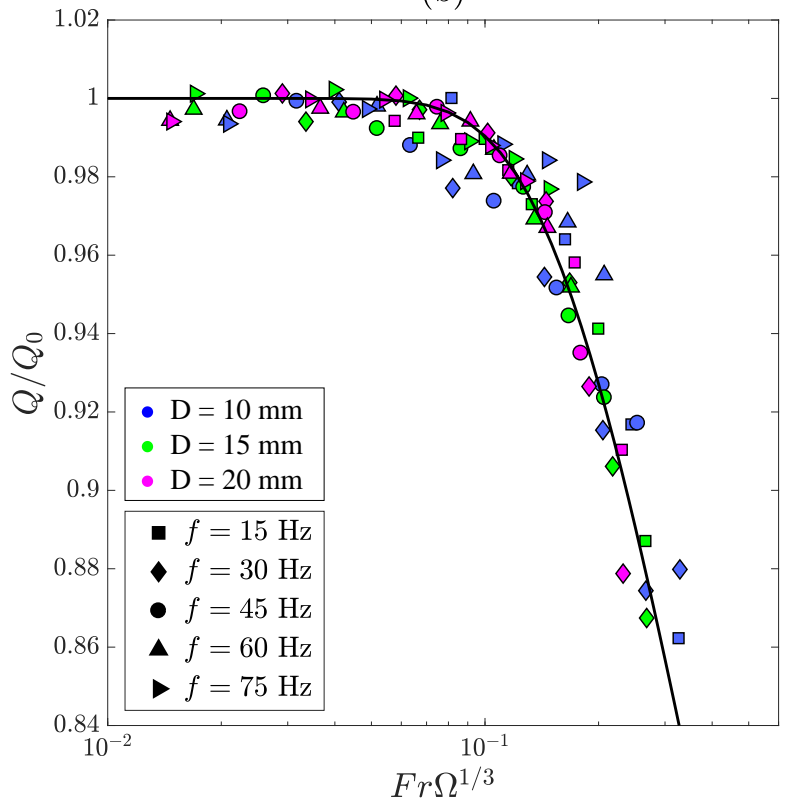

Figure 17: For all $f$ and $D$ values used in simulations. (a) Evolution of the dimensionless flow rate $Q / Q_{0}$ as a function of Fr. Data are fitted to Eq. 7 (solid line), with $a=0.266$ and $F r^{*}=0.399\left(R^{2}=0.869\right)$. Discrepancies appear for high $F r$ values. (b) Evolution of $Q / Q_{0}$ as a function of $F r \Omega^{1 / 3}$. In this case, data are better rationalized and well fitted to Eq. 9 (solid line), with $a_{1}=0.552$ and $b_{1}=0.420\left(R^{2}=0.957\right)$. 


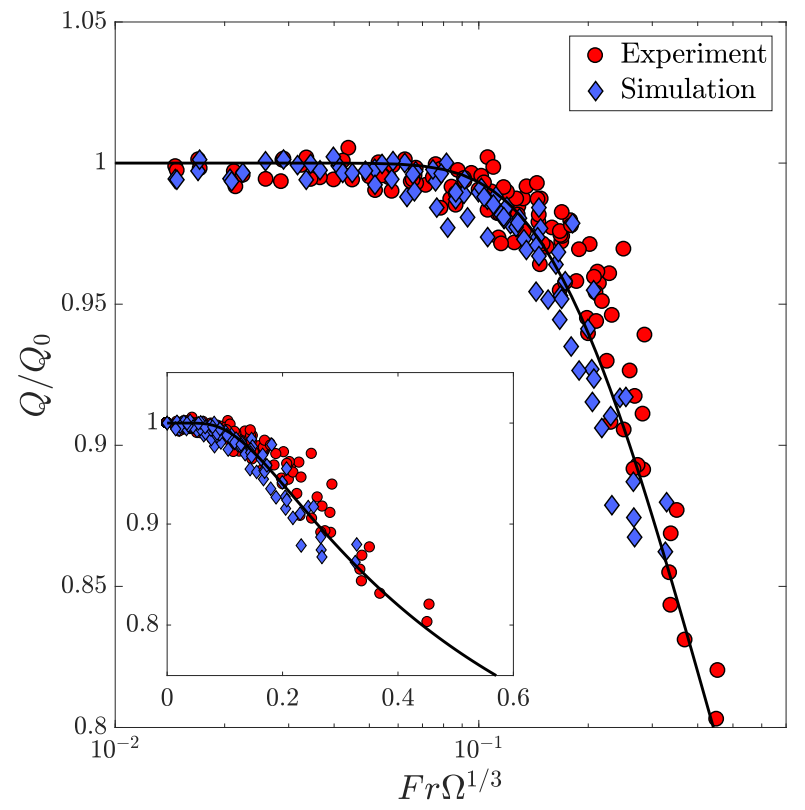

Figure 18: Evolution of $Q / Q_{0}$ as a function of $F r \Omega^{1 / 3}$, for both experimental and numerical results. Data are fitted to Eq. 9 (solid line), with $a_{1}=0.539$ and $b_{1}=0.438\left(R^{2}=0.944\right)$. Insert: linear plot representation.

\subsubsection{Scaling law in the second regime}

From a value of $\operatorname{Fr} \Omega^{1 / 3}$ equals 0.4 , a second regime is observed for which $Q / Q_{0}$ increases with increasing the vibration. The maximum $\mathrm{Fr} \Omega^{1 / 3}$ value reached in experiments is 0.3 (Fig. 17b).

To analyze the flow rate in the second regime, we have chosen to subtract the contribution of the first regime to the normalized flow rate. We define the function $\mathcal{F}_{2}$ as:

$$
\mathcal{F}_{2}=\frac{Q}{Q_{0}}-\mathcal{F}_{1}\left(F r \Omega^{1 / 3}\right)
$$

195

The evolution of $\mathcal{F}_{2}$ as a function of $A$ is plotted in Fig. 19a. In this second regime, the flow rate starts to increase beyond a critical amplitude value that depends on $D$ and $f$, similarly to an "activated process". The critical amplitude value seems to decrease with increasing $f$ and decreasing $D$. This behavior is the fastest for $f=75 \mathrm{~Hz}$ and $D=10 \mathrm{~mm}$, and the slowest for $f=15 \mathrm{~Hz}$ and $D=20 \mathrm{~mm}$ (black arrow in Fig. 19). 
(a)

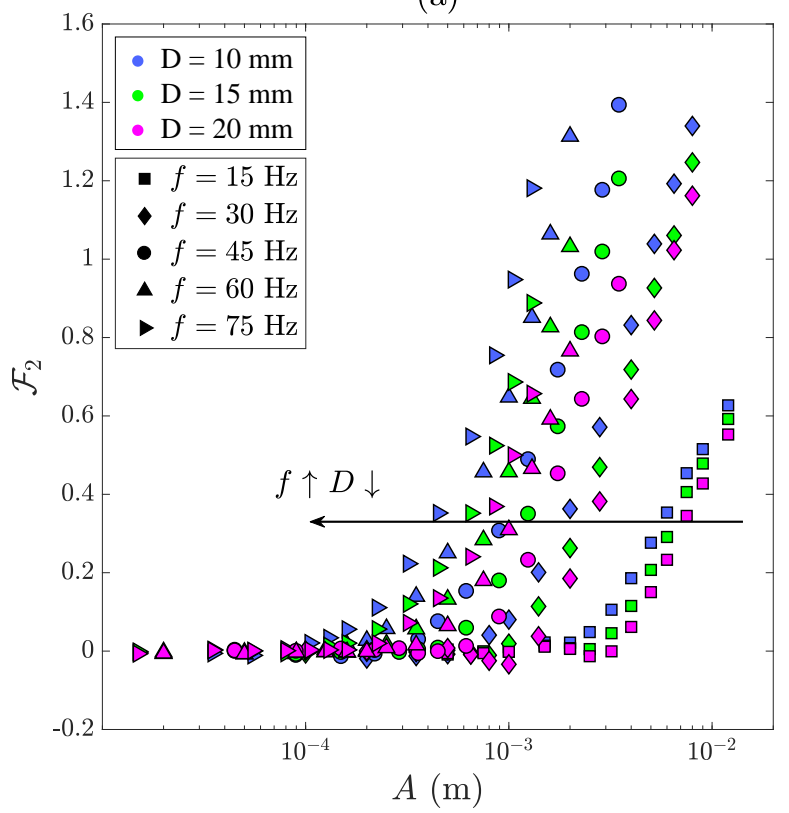

(b)

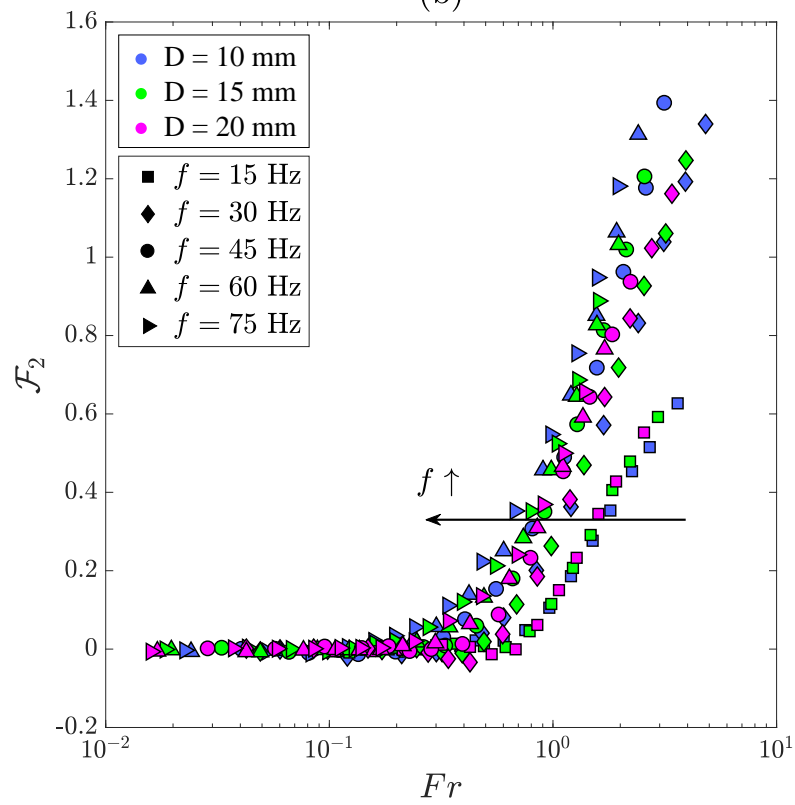

Figure 19: In the second regime and for all $f$ and $D$ values used in simulations: (a) $\mathcal{F}_{2}$ (Eq. 10) as a function of the amplitude $A$. The influence of $f$ and $D$ is denoted by the black arrow. (b) $\mathcal{F}_{2}$ (Eq. 10) as a function of the Froude number $F r$. Only the influence of $f$ is denoted by the black arrow, since the influence of $D$ is taken into account into $F r$.

Regarding Eq. 7 used in the first regime, we propose to fit the data in the second regime to an exponential law, by analogy to an activated process. For high vibration intensity, $\mathcal{F}_{2}$ is expected to tend to a maximum value due to a saturation effect. Therefore, the following equation is proposed:

$$
\mathcal{F}_{2}\left(F r \Omega^{1 / 2}\right)=a_{2} \exp \left(\frac{-b_{2}}{F r \Omega^{1 / 2}}\right)
$$

where $a_{2}$ and $b_{2}$ are two fitting parameters.

The evolution of $\mathcal{F}_{2}$ as a function of the Froude number $F r$ is plotted in Fig. 19b. This scaling seems to take into account the influence of the opening size on the flow rate, but fails to capture the influence of the frequency. As already proposed in the first regime, the relative frequency $\Omega$ is introduced in order to rationalize the data. The best scaling law is obtained for (Fig. 20):

$$
\frac{Q}{Q_{0}}-\mathcal{F}_{1}=\mathcal{F}_{2}\left(F r \Omega^{1 / 2}\right)
$$

Fig. 20 shows the evolution of $\mathcal{F}_{2}$ as a function of $F r \Omega^{1 / 2}$. The data are well fitted to Eq. 11, with $a_{2}=2.41$ and $b_{2}=1.28$. The influence of the relative frequency accounts for the non-linear coupling between vibration-induced energy in flux and losses due to contacts inelasticity $[42,43,44]$. In the first regime, the dissipation takes place mainly in the bulk through intergrain collisions $\left(\Omega^{1 / 3}\right)$, whereas friction at the wall induces a strong dissipation in the second regime $\left(\Omega^{1 / 2}\right)$ as previously suggested in Fig. 15 . An illustration of the effect of $\mathcal{F}_{1}$ and $\mathcal{F}_{2}$ on the flow 
rate as a function of the Froude number is presented in Fig. 21. The entire effect of the vibration is obtained by adding the contribution of each function (Fig. 21, green line).

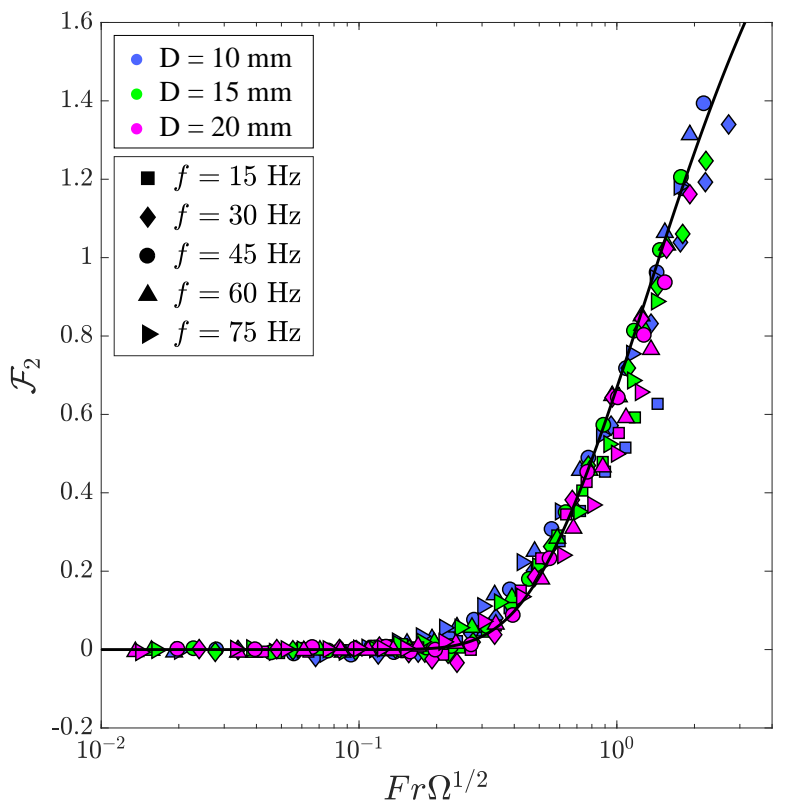

Figure 20: In the second regime, $\mathcal{F}_{2}$ (Eq. 10) as a function of $F r \Omega^{1 / 2}$ for various $f$ and $D$ values used in the simulations. The data well collapse on a master curve that is fitted (solid line) to Eq. 11, with $a_{2}=2.41$ and $b_{2}=1.28\left(R^{2}=0.981\right)$.

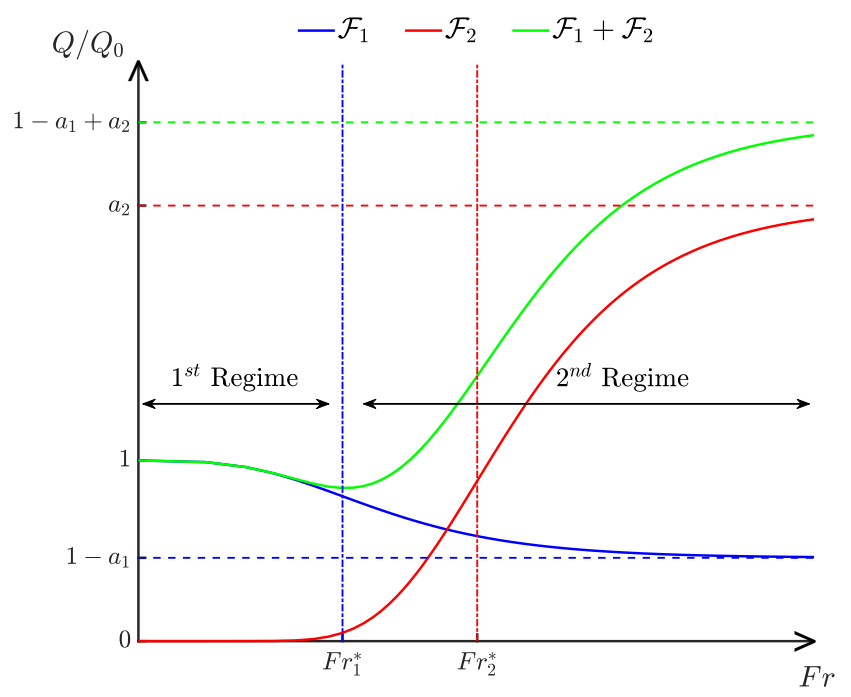

Figure 21: Illustration of the evolution of the dimensionless flow rate as a function of the Froude number for a given relative frequency $\Omega$. The first function $\mathcal{F}_{2}$ (blue line) is an exponential transition from 1 to $1-a_{1}$ around $F r_{1}^{*}=b_{1} / \Omega^{1 / 3}$. The second function $\mathcal{F}_{2}$ (red line) is an exponential transition from 0 to $a_{2}$ around $F r_{2}^{*}=b_{2} / \Omega^{1 / 2}$. The entire effect of the vibration is given by a combination of these two laws (green line). 


\section{Conclusion}

In this paper, we were interested in the discharge of model granular system in a quasi-two dimensional silo. The and numerically. We have put in evidence that adding vibrations makes appear two distinct regimes.

In the first regime, increasing the vibration intensity leads to the decrease of the flow rate. We have shown that this result is explained by the setting in motion, due to vibrations, of particles initially at rest in stagnant areas. Increasing the vibration leads to the decrease of the stagnant areas. In this regime, both experimental and numerical results are in agreement. We have shown the behavior is well captured by an empirical law taking into account the vibration, the opening size and the particle diameter through the Froude number $F r$ and the relative frequency $\Omega$, such as:

$$
\frac{Q}{Q_{0}}=1-a_{1} \exp \left(\frac{-b_{1}}{F r \Omega^{1 / 3}}\right)
$$

In the second regime, increasing the vibration intensity leads to the increase of the flow rate. This regime have been only studied by using simulations, for high vibration frequency value. We have shown that this result is explained by an intermittent burst dynamics due to sudden large rearrangements coming from compression waves propagation all along the packing. We have put in evidence that the behavior is well captured by an empirical law similar to that used in the first regime, such as:

$$
\frac{Q}{Q_{0}}=a_{2} \exp \left(\frac{-b_{2}}{F r \Omega^{1 / 2}}\right)
$$

In a future work, this study could be extended to more complex cases. For instance, by studying the effect of vibrations on non-model particles as cohesive, anisotropic and polydisperse particles. The influence of the silo geometry is also an important parameter to take into account (e.g. different opening angles and 3D cases). Understanding the influence of these parameters on the two proposed laws, and linking them to the local behavior, could be a step towards a general law for granular flows submitted to mechanical vibrations. 


\section{Nomenclature}

\section{Greek letters}

220 $\quad \alpha \quad$ Fitting parameter of the dilatency function $[-]$

$\beta \quad$ Fitting parameter of the dilatency function [-]

$\beta \quad$ Power of the exponential in Weibull model $[-]$

$\Gamma \quad$ Dimensionless acceleration number $[-]$

$\mu_{\text {wall }} \quad$ Wall friction[-]

${ }_{225} \quad \Omega \quad$ Relative frequency $[-]$

$\omega \quad$ Pulsation frequency $\left[t^{-1}\right]$

$\phi_{0} \quad$ Volume fraction at the silo opening [-]

$\phi_{\infty} \quad$ Volume fraction at the center of the silo opening [-]

$\phi_{b} \quad$ Bulk volume fraction $[-]$

230 $\quad \rho \quad$ Particle density $\left[M \cdot L^{-3}\right]$

$\tau \quad$ Characteristic time in Weibull model $[T]$

$\theta \quad$ Opening angle of the silo $[-]$

\section{Latin letters}

$\Delta Q \quad$ Relative difference of flow rate [-]

${ }_{235} \delta Q_{\max }$ Maximum variation of the flow rate [-]

$\mathcal{F} \quad$ Function describing the effect of the vibration on the flow rate based on Wassgren scaling [-]

$\mathcal{F}_{1} \quad$ Function describing the first regime $[-]$

$\mathcal{F}_{2} \quad$ Function describing the second regime $[-]$

$A \quad$ Vibration amplitude $[L]$

a Fitting parameter for the function based on Wassgren scaling [-]

$a_{1} \quad$ Fitting parameter for the first regime $[-]$

$a_{2} \quad$ Fitting parameter for the second regime [-] 
$b_{1} \quad$ Fitting parameter for the first regime $[-]$

$b_{2} \quad$ Fitting parameter for the second regime [-]

${ }_{245} C \quad$ Constant of the flow rate law [-]

$D \quad$ Opening size of the silo $[L]$

$d \quad$ Particle diameter $[L]$

$D_{h} \quad$ Hydraulic diameter of the silo $[L]$

$f \quad$ Vibration frequency $\left[t^{-1}\right]$

250 $F^{*} \quad$ Critical Froude number $[-]$

$G \quad$ Dilatency function at the silo opening [-]

$g \quad$ Gravitational constant $\left[L \cdot t^{-2}\right]$

$H \quad$ Silo height $[L]$

$k \quad$ Constant of the Beverloo law $[-]$

${ }_{255} \quad L \quad$ Silo width $[L]$

$Q \quad$ Flow rate of the silo $\left[M \cdot t^{-1}\right]$

$Q_{0} \quad$ Flow rate without vibration $\left[M \cdot t^{-1}\right]$

$t \quad$ Time $[T]$

$V z \quad$ Relative vertical velocity between the particles and the silo $[L / T]$

$260 \quad W \quad$ Silo depth $[L]$

\section{Acknowledgments}

This study is conducted in the framework of the "PowderReg" project, funded by the European programme Interreg VA GR within the priority axis 4 "Strengthen the competitiveness and the attractiveness of the Grande Région / Großregion". 


\section{Appendix}

The particle properties used in the numerical model are given in Tab. A.1. The values of the friction coefficients are taken from the literature $[45,46]$. Contacts between particles are calculated using a soft sphere model, which imposes a Young's modulus in the range $5 \times 10^{6} \leq E \leq 1 \times 10^{9}$. This approach allows a minimization of the simulation time while giving realistic estimations of the flow rate and flow pattern in a silo, even for particles with a high Young's modulus [47] (for glass beads used in experiments, $E=6.5 \times 10^{10} \mathrm{~Pa}$ ). Various Young's modulus, Poisson's ratio and restitution coefficients are tested to check their influence on the flow behavior, with and without vibration.

(a) Without vibration

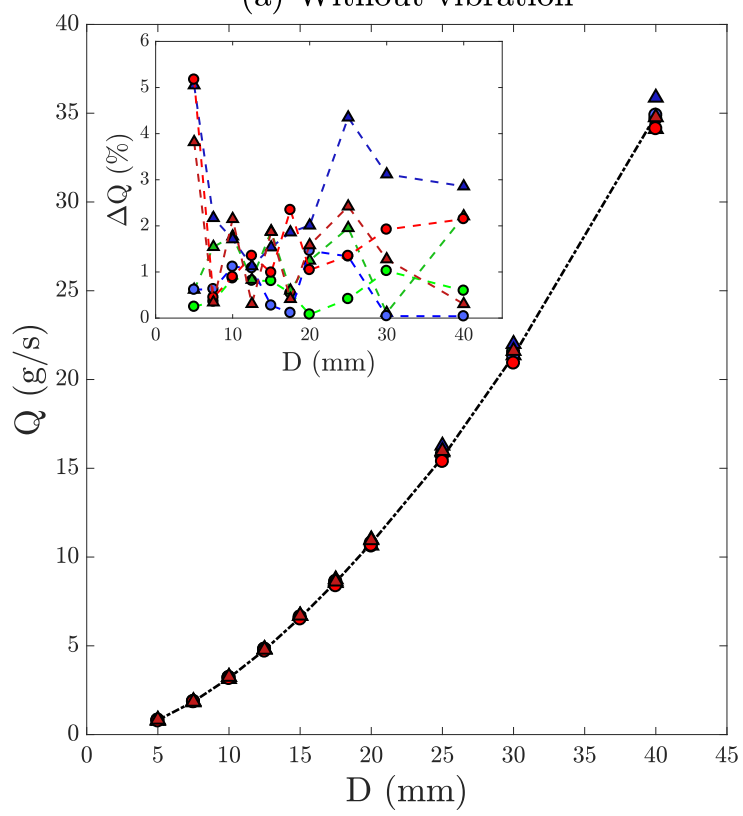

(b) $D=10 \mathrm{~mm}, f=30 \mathrm{~Hz}$

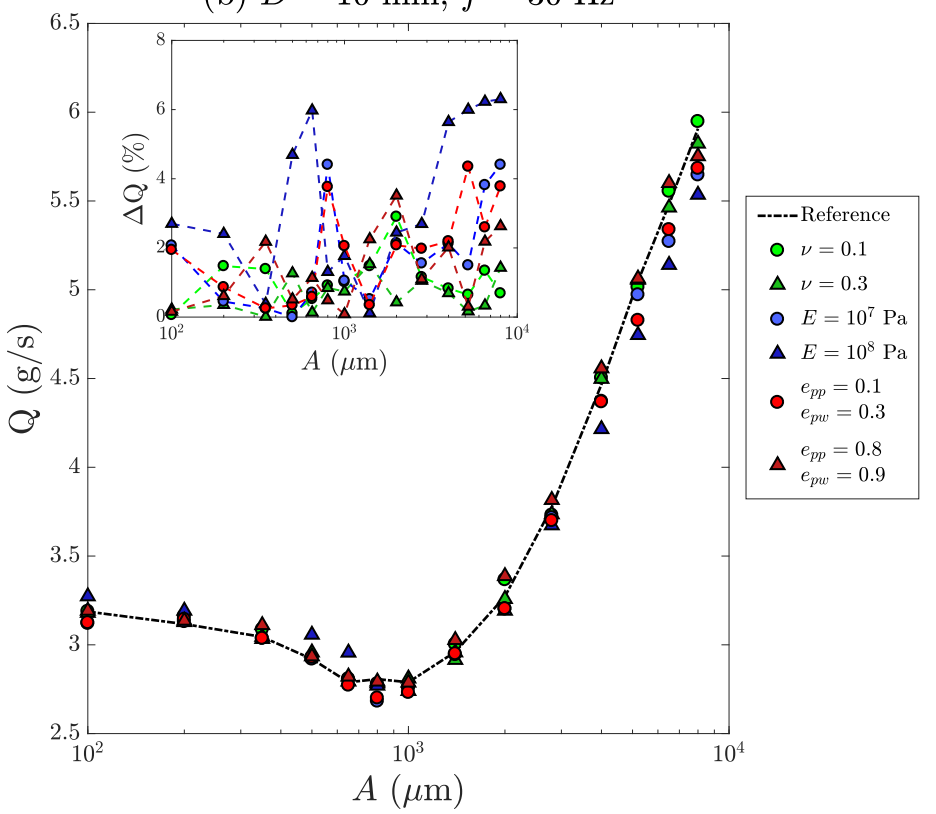

Figure A.1: Flow rate $Q$ obtained by DEM simulation as a function of (a) the opening size $D$, in the case without vibration and (b) the vibration amplitude $A$, at given opening size $(D=10 \mathrm{~mm})$ and frequency $(f=30 \mathrm{~Hz})$. In each case, the reference case (black dotted line) is compared to results obtained with various values of the Young's modulus $E$, the Poisson's ratio $\nu$ and the restitution coefficients (colored markers). Inserts: evolution of the relative difference of flow rate between the reference and a simulated system for each case, given by: $\Delta Q=\left|\left(Q-Q_{\text {reference }}\right) / Q_{\text {reference }}\right|$.

For various coefficients tested, Fig. A.1a and Fig. A.1b show the evolution of the flow rate as a function of the opening size without vibration, and as a function of the amplitude at given opening size and frequency values ( $D=10 \mathrm{~mm}$ and $f=30 \mathrm{~Hz}$ ), respectively. In each case, the influence of the coefficient values is limited to less than $7 \%$ of variation (Fig. A.1, inserts). Therefore, we choose to use the default values proposed in LIGGGHTS.

The equations used in the contact model are described in the following. When the distance between two particles is under the sum of their radius $\left(d_{12}<r_{1}+r_{2}\right)$, the Hertz contact model implemented in the software uses the following equations to calculate the contact force between the particles:

$$
\mathbf{F}_{\mathbf{c}}=\mathbf{F}_{\mathbf{n}}+\mathbf{F}_{\mathbf{t}}
$$


Table A.1: Particle properties used in the simulation. The subscripts $p p$ and $p w$ indicate particle-particle and particle-wall contacts, respectively.

\begin{tabular}{|c|c|c|}
\hline$E$ (Young's Modulus) & & $5 \times 10^{6} \mathrm{~Pa}$ \\
\hline$\nu$ (Poisson's ratio) & & 0.45 \\
\hline \multirow{2}{*}{$\mu$ (Friction coefficient) } & $\mu_{p p}$ & 0.5 \\
\hline & $\mu_{p w}$ & 0.1 \\
\hline \multirow{2}{*}{$e$ (Restitution coefficient) } & $e_{p p}$ & 0.3 \\
\hline & $e_{p w}$ & 0.5 \\
\hline
\end{tabular}

with $\mathbf{F}_{\mathbf{n}}$ the normal force (along the normal vector $\mathbf{n}$ between particle centers) and $\mathbf{F}_{\mathbf{t}}$ the tangential force (along the tangential vector $\mathbf{t}$, perpendicular to $\mathbf{n}$ ), which are given by:

$$
\left.\begin{array}{l}
\mathbf{F}_{\mathbf{n}}=\left(k_{n} \delta_{n}-\gamma_{n} v_{n}\right) \mathbf{n} \\
\mathbf{F}_{\mathbf{t}}=\left(k_{t} \delta_{t}-\gamma_{t} v_{t}\right) \mathbf{t}
\end{array}\right\} \text { with } F_{t} \leq \mu F_{n}
$$

where $k_{n}$ and $k_{t}$ are the normal and tangential contact stiffness, $\gamma_{n}$ and $\gamma_{t}$ are the normal and tangential damping coefficients, $\delta_{n}$ and $\delta_{t}$ are the normal and tangential overlaps, $v_{n}$ and $v_{t}$ are the components of the relative velocity and $\mu$ is the friction between two particles. The contact stiffness and damping coefficients are defined as follows:

$$
\begin{gathered}
k_{n}=\frac{4}{3} E^{*} \sqrt{r^{*} \delta_{n}}, \quad k_{t}=8 G^{*} \sqrt{r^{*} \delta_{n}} \\
\gamma_{n}=-2 \sqrt{\frac{5}{6}} \beta \sqrt{S_{n} m^{*}} \geq 0 \\
\gamma_{t}=-2 \sqrt{\frac{5}{6}} \beta \sqrt{S_{t} m^{*}} \geq 0
\end{gathered}
$$

with $E^{*}$ the effective Young's modulus, $G^{*}$ the effective shear modulus, $r^{*}$ the effective radius, $m^{*}$ the effective mass, $S_{n}$ and $S_{t}$ the damping parameters and $\beta$ the damping ratio. The effective values are calculated from the properties of the two particles in contact, using the following equations:

$$
\begin{gathered}
\frac{1}{E^{*}}=\frac{1-\nu_{1}^{2}}{E_{1}}+\frac{1-\nu_{2}^{2}}{E_{2}} \\
\frac{1}{G^{*}}=\frac{2\left(2-\nu_{1}^{2}\right)\left(1+\nu_{1}\right)}{E_{1}}+\frac{2\left(2-\nu_{2}^{2}\right)\left(1+\nu_{2}\right)}{E_{2}} \\
\frac{1}{r^{*}}=\frac{1}{r_{1}}+\frac{1}{r_{2}}, \frac{1}{m^{*}}=\frac{1}{m_{1}}+\frac{1}{m_{2}}
\end{gathered}
$$

Lastly, the damping parameters and damping ratio are defined as follows:

$$
\begin{gathered}
S_{n}=2 E^{*} \sqrt{r * \delta_{n}}, \quad S_{t}=8 G^{*} \sqrt{r^{*} \delta_{n}} \\
\beta=\frac{\ln (e)}{\sqrt{\ln ^{2}(e)+\pi^{2}}}
\end{gathered}
$$




\section{References}

[1] M. Fayed, L. Otten, Handbook of powder science \& technology, Springer Science \& Business Media, 2013.

[2] W. A. Beverloo, H. A. Leniger, J. Van de Velde, The flow of granular solids through orifices, Chemical engineering science 15 (3-4) (1961) 260-269.

[3] R. Nedderman, U. Tüzün, S. Savage, G. Houlsby, The flow of granular materials-i: Discharge rates from hoppers, Chem. Eng. Sci 37 (11) (1982) 1597-1609.

[4] C. Mankoc, A. Janda, R. Arevalo, J. Pastor, I. Zuriguel, A. Garcimartín, D. Maza, The flow rate of granular materials through an orifice, Granular Matter 9 (6) (2007) 407-414.

[5] A. Janda, I. Zuriguel, D. Maza, Flow rate of particles through apertures obtained from self-similar density and velocity profiles, Physical review letters 108 (24) (2012) 248001.

[6] M. Benyamine, P. Aussillous, B. Dalloz-Dubrujeaud, Discharge flow of a granular media from a silo: effect of the packing fraction and of the hopper angle, in: EPJ Web of Conferences, Vol. 140, EDP Sciences, 2017, p. 03043.

[7] W. R. Ketterhagen, J. S. Curtis, C. R. Wassgren, B. C. Hancock, Modeling granular segregation in flow from quasi-three-dimensional, wedge-shaped hoppers, Powder Technology 179 (3) (2008) 126-143.

[8] A. Anand, J. S. Curtis, C. R. Wassgren, B. C. Hancock, W. R. Ketterhagen, Predicting discharge dynamics of wet cohesive particles from a rectangular hopper using the discrete element method (dem), Chemical Engineering Science 64 (24) (2009) 5268-5275.

[9] D. Hund, D. Weis, R. Hesse, S. Antonyuk, Simulation study of the discharge characteristics of silos with cohesive particles, in: EPJ Web of Conferences, Vol. 140, EDP Sciences, 2017, p. 08015.

[10] K. To, P.-Y. Lai, H. Pak, Jamming of granular flow in a two-dimensional hopper, Physical review letters 86 (1) (2001) 71.

[11] I. Zuriguel, A. Garcimartín, D. Maza, L. A. Pugnaloni, J. Pastor, Jamming during the discharge of granular matter from a silo, Physical Review E 71 (5) (2005) 051303.

[12] A. Janda, I. Zuriguel, A. Garcimartín, L. A. Pugnaloni, D. Maza, Jamming and critical outlet size in the discharge of a two-dimensional silo, EPL (Europhysics Letters) 84 (4) (2008) 44002.

[13] R. O. Uñac, A. M. Vidales, O. Benegas, I. Ippolito, Experimental study of discharge rate fluctuations in a silo with different hopper geometries, Powder technology 225 (2012) 214-220.

[14] P. W. Cleary, M. L. Sawley, Dem modelling of industrial granular flows: 3d case studies and the effect of particle shape on hopper discharge, Applied Mathematical Modelling 26 (2) (2002) 89-111. 
[15] D. Höhner, S. Wirtz, V. Scherer, A study on the influence of particle shape on the mechanical interactions of granular media in a hopper using the discrete element method, Powder technology 278 (2015) 286-305.

[16] N. Govender, D. N. Wilke, P. Pizette, N.-E. Abriak, A study of shape non-uniformity and poly-dispersity in hopper discharge of spherical and polyhedral particle systems using the blaze-dem gpu code, Applied Mathematics and Computation 319 (2018) 318-336.

[17] C. Mankoc, A. Garcimartín, I. Zuriguel, D. Maza, L. A. Pugnaloni, Role of vibrations in the jamming and unjamming of grains discharging from a silo, Physical Review E 80 (1) (2009) 011309.

[18] I. Zuriguel, Á. Janda, R. Arévalo, D. Maza, Á. Garcimartín, Clogging and unclogging of many-particle systems passing through a bottleneck, in: EPJ Web of Conferences, Vol. 140, EDP Sciences, 2017, p. 01002.

[19] B. Guerrero, C. Lozano, I. Zuriguel, A. Garcimartín, Dynamics of breaking arches under a constant vibration, in: EPJ Web of Conferences, Vol. 140, EDP Sciences, 2017, p. 03016.

[20] C. Zhang, C. Qiu, C. Pu, X. Fan, P. Cao, The mechanism of vibrations-aided gravitational flow with overhanging style in hopper, Powder technology 327 (2018) 291-302.

[21] T. Kollmann, J. Tomas, Effect of applied vibration on silo hopper design, Particulate science and technology 20 (1) (2002) 15-31.

[22] F. Y. Fraige, P. A. Langston, A. J. Matchett, J. Dodds, Vibration induced flow in hoppers: Dem 2d polygon model, Particuology 6 (6) (2008) 455-466.

[23] S. K. de Richter, G. Le Caër, R. Delannay, Heterogeneous dynamics of a granular pack under vertical tapping, EPL (Europhysics Letters) 85 (5) (2009) 58004.

[24] C. Hanotin, P. Marchal, L. J. Michot, C. Baravian, S. K. de Richter, Dynamics of vibrated granular suspensions probed by mechanical spectroscopy and diffusing wave spectroscopy measurements, Soft Matter 9 (39) (2013) 9352-9360.

[25] N. Gaudel, S. K. de Richter, N. Louvet, M. Jenny, S. Skali-Lami, Bulk and local rheology in a dense and vibrated granular suspension, Physical Review E 96 (6) (2017) 062905.

[26] N. Gaudel, S. K. de Richter, N. Louvet, M. Jenny, S. Skali-Lami, Granular avalanches down inclined and vibrated planes, Physical Review E 94 (3) (2016) 032904.

[27] M. Hunt, R. Weathers, A. Lee, C. Brennen, C. Wassgren, Effects of horizontal vibration on hopper flows of granular materials, Physics of fluids 11 (1) (1999) 68-75.

[28] C. R. Wassgren, M. L. Hunt, P. Freese, J. Palamara, C. Brennen, Effects of vertical vibration on hopper flows of granular material, Physics of Fluids 14 (10) (2002) 3439-3448. 
[29] P. A. Langston, U. Tüzün, D. M. Heyes, Discrete element simulation of granular flow in 2d and 3d hoppers: dependence of discharge rate and wall stress on particle interactions, Chemical Engineering Science 50 (6) (1995) 967-987.

[31] T. G. Drake, Granular flow: physical experiments and their implications for microstructural theories, Journal of Fluid Mechanics 225 (1991) 121-152.

[32] A. Medina, J. Cordova, E. Luna, C. Trevino, Velocity field measurements in granular gravity flow in a near 2d silo, Physics Letters A 250 (1-3) (1998) 111-116.

[33] A. Janda, D. Maza, A. Garcimartín, E. Kolb, J. Lanuza, E. Clément, Unjamming a granular hopper by vibration, EPL (Europhysics Letters) 87 (2) (2009) 24002.

[34] T. Van De Laar, S. Ten Klooster, K. Schroën, J. Sprakel, Transition-state theory predicts clogging at the microscale, Scientific reports 6 (1) (2016) 1-8.

[35] H. Rinne, The Weibull distribution: a handbook, Chapman and Hall/CRC, 2008.

[36] J. M. Pastor, A. Garcimartín, P. A. Gago, J. P. Peralta, C. Martín-Gómez, L. M. Ferrer, D. Maza, D. R. Parisi, L. A. Pugnaloni, I. Zuriguel, Experimental proof of faster-is-slower in systems of frictional particles flowing through constrictions, Physical Review E 92 (6) (2015) 062817.

[37] J. Crassous, J.-F. Metayer, P. Richard, C. Laroche, Experimental study of a creeping granular flow at very low velocity, Journal of Statistical Mechanics: Theory and Experiment 2008 (03) (2008) P03009.

[38] C. Goniva, C. Kloss, A. Hager, S. Pirker, An open source cfd-dem perspective, in: Proceedings of OpenFOAM Workshop, Göteborg, 2010, pp. 1-10.

[39] C. Kloss, C. Goniva, A. Hager, S. Amberger, S. Pirker, Models, algorithms and validation for opensource dem and cfd-dem, Progress in Computational Fluid Dynamics, an International Journal 12 (2-3) (2012) 140-152.

[40] P. A. Cundall, O. D. Strack, A discrete numerical model for granular assemblies, geotechnique 29 (1) (1979) $47-65$.

[41] B. Andreotti, Y. Forterre, O. Pouliquen, Granular media: between fluid and solid, Cambridge University Press, 2013.

[42] I. S. Aranson, L. S. Tsimring, Patterns and collective behavior in granular media: Theoretical concepts, Reviews of modern physics 78 (2) (2006) 641.

[43] E. Aharonov, D. Sparks, Rigidity phase transition in granular packings, Physical Review E 60 (6) (1999) 6890. 
[44] F. Melo, P. Umbanhowar, H. L. Swinney, Transition to parametric wave patterns in a vertically oscillated granular layer, Physical review letters 72 (1) (1994) 172.

[45] J.-C. Géminard, W. Losert, Frictional properties of bidisperse granular matter: Effect of mixing ratio, Physical Review E 65 (4) (2002) 041301.

[46] A. Lorenz, C. Tuozzolo, M. Louge, Measurements of impact properties of small, nearly spherical particles, Experimental Mechanics 37 (3) (1997) 292-298.

[47] Y. Xu, K. Kafui, C. Thornton, G. Lian, Effects of material properties on granular flow in a silo using dem simulation, Particulate Science and Technology 20 (2) (2002) 109-124. 